\title{
Een devoet epistel ghescreven enen koc
}

door

\author{
Dr. LOUIS DE MAN
}

\section{HET HANDSCHRIFT}

$\mathrm{B}^{\mathrm{a}} 75$ is de signatuur van een hs. dat berust in de Erzbischöfliche Bibliothek te Paderborn en dat twee teksten bevat met raadgevingen en beschouwingen van geestelijke aard. De eerste, het epistel voor een kloosterkok, wordt beneden uitgegeven; de tweede, een fragment, komt later aan de beurt.

Het hs. zit gebonden in een moderne kartonnen band met lederen rug, waarop in goud de signatuur werd aangebracht.

Modern is ook het schutblad voor- en achteraan; op het voorste staat van een 19de-eeuwse hand Geschrieben anno 1455, Betrachtungsbuch für einen Klosterkoch.

Het eerste deel telt 39 folio's, in perkament of in papier, $10 \mathrm{~cm}$ breed $\times 14 \mathrm{~cm}$ hoog; hierbij werd fol. 1, dat slechts een talon is, afgerekend en werd fol. 17, dat uitgescheurd werd, meegeteld. Dit deel beslaat vier katernen.

Het eerste katern bevat 9 folio's; het eerste folium werd gereduceerd tot een talon van $2,5 \mathrm{~cm}$ breed. Fol. 1-2; 5-6 en 9-10 zijn van perkament, 3-4 en 7-8 van papier. Het tweede katern bestaat uit 12 folio's; hiervan zijn $2,3,4,5,9$ en 10 van papier; 8 is uitgescheurd. Het derde heeft 14 folio's en hiervan zijn $1,7,8$ en 14 van perkament. Het vierde katern ten slotte bedraagt vier perkamenten folio's.

In dit eerste deel komen reklamen, kustoden of foliëring niet voor; op de papieren folio's is liniëring, door de band voor 20 of 21 regels.

Verschillende handen hebben aan deel I meegeschreven; het schrift bleef daarbij de littera textualis, met op sommige folio's soms de bastarda. Ieder begin van een kapittel werd versierd met rode lombarden; over het algemeen heeft dan de daarop volgende letter het formaat van een majuskel die met zwarte inkt getekend werd.

Naast of in de lombarden staan de kleine representanten die op de meeste plaatsen nog zichtbaar zijn.

In de tekst zelf wordt een nieuwe zin aangeduid door een majuskel waarvan het eerste streepje rood is. Rood zijn ook de vol- 
gende titels boven de kapittels: Hir begbint... (2 r); Woe een goetwillich koc... (2 v); Woe be hem hebben sal... (5 v); Wo dat be nicht beswart... (12 v); Wo be hem sal hebben... (16 r); Woe dat men nicht altoes... (21 r); Wo be hem oefenen sal... (27 v).

Met rode inkt ten slotte werden ook een paar verbeteringen in de tekst aangebracht : zie tekst.

De bladspiegel van het hs. is gaaf behalve op fol. 37 waar, bij het neerschrijven, rekening werd gehouden met een fout in het perkament; onderaan de folio's is het stuk gehavend door vocht.

Op fol. 39 v staat de datum : Ghescreuen jnt jaer vns beren dusent $c c c c^{\circ}$ unde .lv.; aan de hand van de verschrijvingen in de tekst mag men wel besluiten dat hij een kopie is uit het jaar 1455 en niet de oorspronkelijke versie.

\section{DE TITEL}

De titel luidt «Hir beghint een deuoet epistel ghescreuen enen koc in eenen reguler closter $\gg$. Daarbij valt het beroep van de begunstigde op. Gaat men in de litteratuur zoeken naar de sociale en eventueel culturele functie van deze kok, dan komt men via een of andere index wel terecht in de middeleeuwse keuken of bij de zgn. keukentaal, maar de gegevens beperken zich tot eetgerei, recepten of gastronomie. De typologie van de middeleeuwse kok werd tot nog toe nog niet grondig bestudeerd, maar de volgende aanduidingen mogen voor ons opzet volstaan.

E.R. Curtius legt de nadruk op het feit dat de kok in de litteratuur van de laat-Romeinse tijd en in de vroege middeleeuwen een komisch, belachelijk personage is, iemand die bespot wordt om zijn voorkomen dat door rook zwart geworden is. De Trierse kloosterleraar Winrich vertelt (ca. 1070), al klagend, hoe hij naar de keuken verplaatst werd : het blijkt een echte degradatie te zijn.

Uit de Romeinse comedie werd de kok een «vilissimum mancipium », dé bron van humor in de middeleeuwse letterkunde ${ }^{1}$.

L. Mackensen voegt hieraan toe dat de geestelijke dichter lacht met de geborneerdheid van de keukenbroeder ${ }^{2}$; hij wijst ook terecht op het feit dat de Nibelungendichter, midden in de tragedie, nog eens hartelijk doet lachen door het beeld van de vluchtende kokjongens, in een wirwar van potten en pannen ${ }^{3}$.

(1) Europäische Literatur und Lateinisches Mittelalter (Bern, 19613), blz. 431434 (Kap. "Küchenhumor und andere Ridicula").

(2) $Z u$ Walthers Spiessbratenspruch (= Studien zur deutschen Philologie des Mittelalters Friedrich Panzer zum 80. Geburtstag am 4. September 1950 dargebracht. Herausgeg. von R. Kienast. Heidelberg, 1950), blz. 48-49.

(3) Ibid., blz. 49. 
Het klassieke beeld van de bespottelijke kok in de ridderpoëzie is Rumolt: «Für den mittelalterlichen Hörer ist Rumolts Wesensart durchaus eindeutig durch seinen Beruf bestimmt, was er sagt, ist ohnehin 'komisch'. Und der Dichter hat die Gewissheit, dass Hörer und Leser ohne grosses Bedenken begreifen, was mit 'Rumolts Rat' gemeint ist ${ }^{4}$. De berookte kok is de man die bescheiden werk verricht en op de laagste trap van de culinaire hiërarchie staat; tussen hem en de gasten staan nog de spisedrager (dapifer) en de schenker (pincerna). De toehoorders lachen wanneer verteld wordt van de $z w a a n$ (of is het een gans ?) die eens op het water zwom (Olim lacus colueram) en die nu, aan een braadspit, boven het vuur hangt (Girat, regirat garcifer), - niet omdat vlees gebraden wordt, maar omdat zij, in hun verbeelding, een zwarte kok zien opereren ${ }^{5}$.

Het beeld van de "cocus niger 》 die weinig fijn is en vastzit in zijn berookte keuken, lijkt aanvaardbaar ${ }^{6}$. Van minder belang hier is de nieuwe voorstelling van de kok, die Curtius vermeldt : «Im Rainouart-Epos wird endlich der derbkomische Küchenjunge zum epischen Helden. Die Verbindung von Küche und Kriegertum scheint übrigens auch in Wirklichkeit vorgekommen zu sein ${ }^{7}$. Ook Mackensen snijdt het probleem aan : «Rumolts Gegenstück ist Wolframs Rennewart, auch er, solange er Küchenjunge ist, eine komische Gestalt. Das Anliegen Wolframs an die Rennewartgestalt ist es doch wohl zu zeigen, wie aus einem vilain, aus einem Küchentölpel, ein echter Ritter wird » ${ }^{8}$.

De overgang van weinig geleerd tot verfijnd bestaat op het materieel plan; niets verzet zich tegen de bewering dat hier en daar, in gunstige omstandigheden, een kloosterkok zijn onwetendheid heeft afgelegd en nu zijn meditaties dicteert of zelf schrijft.

Het is daarom niet nodig te grijpen naar de bijbelse belofte, de kleinen te verheffen, of de autoriteit van het Loflied in te roepen ${ }^{9}$.

(4) Ibid.

(5) De citaten stammen uit de "Carmina Burana" (hs. 13e eeuw, liederenverzameling uit het klooster Benediktbeuren); zie de tekst met Duitse vertaling in Carmina Burana, Lieder der Vaganten, Lateinisch und Deutsch nach $L$. Laistner herausgeg. von E. Brost (Heidelberg, 1956), blz. 50-53; de liederen werden ook door C. Orff, een bekend Duits komponist, in 1937 bewerkt. De oorspronkelijke titel luidde hier : Cantiones profanae cantoribus et choris cantandae comitantibus instrumentis atque imaginibus magicis. Orffs Carmina, op plaat opgenomen door de Deutsche Grammophon Gesellschaft LPM [M 33], bevatten ook een tekstboek met kokgeschiedenis (zie nr. 12). Het koklied van Orff is overigens prachtige persiflage.

(6) Vgl. ook later nog het beeld van kok en keuken in S. Brants Narrenscbiff (uitg. Fr. Zarncke, Leipzig, 1854), blz. 78-79.

(7) Op. cit., blz. 432.

(8) Op. sit., blz. 50.

(9) Zie tekst en vertaling in M.M. Davy, Guillaume de Saint-Thierry, Commentaire sur le cantique des cantiques (Parijs, 1958) (= Bibl. des Textes Philosophiques), blz. 54 en 68. 
Interessanter voor ons opzet is de "nauwe verbondheid 》 tussen het gebedsleven en handenarbeid die in mannen- en vrouwenkloosters bestond ${ }^{10}$. Handenarbeid kan ten andere in een klooster opgelegd worden om de nederigheid op de proef te stellen; dit was het geval met Th. van Stamheyn die, in 1396, op een boerderij te Almelo moest werken vóór hij nog kok werd te Deventer ${ }^{11}$. En te Almelo had hij als metgezel Hendrik Kindeshof, ook een kok, die later de eerste prior zou worden (1394-1399) van het klooster te Frenswegen ${ }^{12}$.

Het Frensweger handschrift citeert «den guede (= goederen) Iohannes Kessels oers cockes, die in der werlt seer rijck was ${ }^{13}$ en noemt hem elders «die oetmoedighe cock» ${ }^{14}$.

De oversten konden tot keukenarbeid verplichten, maar gaven ook zelf het voorbeeld; misschien behoort Kessel tot de groep die ook «om oefeninghe » het werk in de keuken volbracht, en dit « voorrecht » behield. Men vergelijke volgend citaat : « Die clercken fratres (des huses Sunte Iheronimusberch bij Hattum in die Velue) deden ducke... blidelike ende vroelike die oetmoedighe ende grove werke uut noetsaken of om oefeninghe, als in der coken die potte, scotelen ende andere dinghe vlitelic te wasschen, somtijt in den bachues broet te maken of in den brouhues helpen te brouwen ${ }^{15}$.

Theodericus van Kueck, uit het klooster te Albergen, «enen clerck die te Zwolle hadde ter scholen ghegaen », doet keukenwerk: « Hie cokede, hie backede, hie was brouwer, hie slachtede, hie was gaerdenaer, hie zeyede daer yn manigherleye saet tot cruden, hie wan dat moescruet ende bereidet ten etene $\gg{ }^{16}$.

Van Heer Florens, «den iersten rector ende pater der vergadderinghen priesteren ende clercken toe Deventer 》 wordt verteld dat hij " hielt... oec sine weke in der coken nae sinen vermoghen ${ }^{17}$ en in die week, zei hij, " hope (ic) meerre vrucht te vercrighen van der anderre ghebeden die in der kerken sin dan of ic alleen bedede $\gg{ }^{18}$. Hij wijst hierbij op de verdiensten van het gemeen-

(10) St. Axters, Geschiedenis van de vroombeid in de Nederlanden II (Antwerpen, 1953), blz. 322 ; III (Antwerpen, 1956), blz. 106.

(11) C. van der Wansem, Het ontstaan en de geschiedenis der Broederschat van bet Gemene Leven tot 1400 (Leuven, 1958) (= Univ. te Leuven, Publicaties op het gebied der geschiedenis en der philologie, 4e reeks, dl. 12), blz. 139-140.

(12) Ibid., - Vgl. de tekst over Hendrik Kindeshof in W. Jappe Alberts en A.L. Hulshoff, Het Frensweger bandschrift (Groningen, 1958) (= Rijksuniv. Utrecht. Teksten en Documenten I), blz. 38.

(13) W. Jappe Alberts en A.L. Hulshoff, op. cit., blz. 76.

(14) Ibid., blz. 47.

(15) Ibid., blz. 203

(16) Ibid., blz. 239.

(17) Ibid., blz. 38 .

(18) Ibid., blz. 38-39 
schappelijk gebed, verdiensten die in ons handschrift niet worden aangeraakt.

Heer Amilius, de opvolger van Florens, spaarde zich niet «mer uut ghehoersamheit angheng hy oec swaren arbeit, te backen, te brouwen " ${ }^{19}$. Hij leerde dat " een yeghelic sal bereyt wesen tot een yegheliken ambochte dat hem opgelecht wort, het sy groet of cleyn, als brouwen, backen, of dat pargament te bewaren ${ }^{20}$.

Bij herhaling wordt erop gewezen dat de nederigheid, gepaard met echte vroomheid, beloond wordt.

$\mathrm{Er}$ is het «exempel van eynre hilliger ionferen 》 die uit ootmoed naar het klooster gaat en er "all dat onreynste, snoyste werk ynder koeken » doet; bij de medezusters geldt ze als «die is geeck ${ }^{21}$, - maar ze wordt verheven.

«Suster elsken... ten diepenveen » «plach vaeke in der coeken te wesen » en er te werken. Met haar gebeurde een mirakel ${ }^{22}$.

«Suster dayken verwaerde een wijltijdes dat reventer ende diende den susteren ter tafelen ». $\mathrm{Zij}$ werd later «schoelmeysterssche » en ten slotte priorin ${ }^{23}$.

Keukendienst of bereiden van eten in een middeleeuws klooster kon gelden als uitstekend werk : het was een toevlucht voor de ootmoedigen en een toetssteen voor de gehoorzaamheid. Het is opvallend hoe vaak in de verhalen het accent ligt op het feit dat een kok het kon brengen tot hogere functies. De herhaling wijst op een systeem en op een pedagogische bedoeling.

Wie over de kok in de middeleeuwen spreekt, denkt aan Jan van Leeuwen, de leerling van Ruusbroec; hij heet «primus coquus hujus monasterii (i.e. Groenendaal) », in de volksmond «de Goede Kok ». Hij was ook werkelijk verantwoordelijk voor keuken en kleedkamer ${ }^{24}$. Uit het bestaan van een aantal hss. met excerpten uit Van Leuwen mag men afleiden dat zijn succes tot het eind van de 15 e eeuw groot was ${ }^{25}$. Dit succes volstaat om in geestelijke

(19) Ibid., blz. 53.

(20) Ibid., blz. 56.

(21) Exempel uit de Spegel der Susteren, zie C.G.N. de Vooys, Middelnederlandse sticbtelijke exempelen (Zwolle, 1953) (= Zwolse drukken nr. 1), blz. 13-14.

(22) D.A. Brinkerink, Van den doechden der vuriger ende stichtiger susteren van Diepenveen (Hs. D) (Leiden, z.j.) (= Bibliotheek van Mnl. Letterkunde), blz. 298.

(23) Ibid., blz. 360.

(24) Zie St. Axters, op. cit., II, blz. 297-298. - In het Boec van der bedinghen vertelt Van Leeuwen dat hij dagelijks op het gestelde uur moest klaar zijn met het eten van dertig kloosterlingen, ibid., blz. 306.

(25) Ibid., II, blz. 317. - Ook later is dit nog zo; Pieter Croon publiceert in 1663 een ,allegorie van het keukenbedrijf", nl. Cocus bonus ofte Geestelijcke bemerckinge op de tafel ende spysen van een volkommen maeltyt, voorafgegaan door een biografie van Van Leeuwen, ibid., II, blz. $318,-\mathrm{Bij}$ W. de Vreese komt de titel voor van een andere uitgave, (nl. Antwerpen, 1665) : Cocus Bonus ofte Geestelijcke 
werken de formule of de mode te verklaren van een kok als leraar.

Zonder twijfel hebben andere factoren bijgedragen tot de verspreiding. Zo is er bv. de uit de 15 e eeuw overgeleverde legende van "Christus, de geestelijke kok », die ouder moet zijn dan de bewaarde versie, al gaat ze terug op een parallellegende, waarin de kok niet verschijnt ${ }^{26}$.

Deze Christus-kok-legende verhaalt hoe, met Pasen, twaalf jonkvrouwen te communie gaan. Daarna willen ze in hun kluis eten. De eerste gaat daarom naar de keuken; omdat ze niet terugkeert, gaat de tweede, en zo voort, tot de laatste. In de keuken is Christus, de geestelijke kok. De twaalf hebben hem gevraagd wie hij was en de openbaring is zo overweldigend dat iedere jonkvrouw 《truncken von dem edlen cypper win göttlicher liebin 》 neervalt ${ }^{27}$.

Christus in de gedaante van een kok in de keuken, onderwijst. Het beeld stemt overeen met andere voorstellingen, waarin Christus ook spijs en drank geeft. Men vgl. een 15de-eeuwse tekst uit Brussel : «Ons lieue h(er)e Jhs xps leerde eene(n) van sine vru(n) den .xv. Pater $n$ (oste) $r$ ende .xv. Aue maria »; « ic wil hem selue .vij. daghe spisen... met minen heyleghe lichame voer den eeweliken dorst ende hongher. Ende ic wil hem drinken gheuen met myn heylich bloet voer den eeweliken dorst» $(1 \mathrm{r})$; «Ende als icken daer hebbe ghebracht (i.e. in hemelrike) So willic hem daer schincke sunderlinghe(n) dranc wten borne mynder godheyt » $(1 \mathrm{v}){ }^{28}$. Naar de geest stemt deze kokgedaante overeen met andere naïeve en vaak zo realistische verhalen, bv. met Christus' Hart als de Geestelijke Taveerne : "Comt alle drincken : / U sal scincken / Ihesus goet / Ute desen Tappe, / Met vollen nappe / Sijns herten bloet ${ }^{28}$.

Het pas geciteerd 《truncken » zijn van «liebin» is daarbij te vergelijken met het hier voorkomende «Ghi selt ontfuncken / Ende werden druncken / Van minnen sciere ${ }^{30}$.

De curieuze voorstellingen van Christus als kok of van de Geestelijke Taveerne kunnen aangevuld worden door een laatste illustratie van maniërisme, $\mathrm{nl}$. door een citaat van Angelus Silesius : $\ll \operatorname{Er}[=$ Jesus $]$ setzet ihnen ferner vor / die Klarheit seiner Seele

sinnebeelden ende Godvruchtige Uyt-legginghen $O p$ alle de ghereetschap van den Kock (in: Bijdragen tot de kennis van het leven en de werken van Jan van Ruusbroec. Gent, 1896, blz. 33.

(26) O. Weinrich, Triskaidekadiscbe Studien (Giessen, 1916) (= Religionsgeschichtliche Versuche und Vorarbeiten XVI, 1), blz. 61.

(27) Ibid., blz. 62 .

(28) Mss. div. 3986, ARA Brussel ; hierin zitten verschillende resten van hss., o.a. een perkamenten fragment (vier bladjes, 15e e.) waaruit bovenstaande citaten stammen.

(29) M. Meertens, De godsvrucht in de Nederlanden II (Brussel, 1931) (= Leuvense Studieën en Tekstuitgaven), blz. 31.

(30) Ibid. 
/ Und hebt sie etwas mehr empor / In der durchlauchten Höhle; / Zuletzt lässt er das Konfekt / Von seiner Gottheit kommen / Da bleibt die Tafel stets bedeckt / Und wird nie abgenommen ${ }^{31}$. Gerugsteund worden de verhalen door de woorden van de consecratie en de postcommunio.

In ons hs. is de kok de bescheiden figuur gebleven die met raad en troost wordt bijgestaan.

\section{DE INHOUD}

I. Inleiding

a) De inleiding begint met een aanroeping of invocatio : « $\mathrm{DE}$ vrede godes de alle synnen bouen ghaet de mod behoden vnde bewaren iv herte vnde yuwe synne vnde mote yuwe verstandicheit verclaren to synen loue vnde to syner eeren in vnsen leuen heren ihesum christum ».

b) Het daaropvolgend «Leue broder in vnsen leuen heren » is, hoe vaag en traditioneel ook, te beschouwen als een inscriptio: de bestemmeling wordt aangesproken.

c) De aanleiding tot het schrijven van de tekst wordt tamelijk breedvoerig uiteengezet; het is een soort van narratio : vermits van mij wordt verwacht dat ik schrijf over het probleem van de gehoorzaamheid tegenover de oversten, zal ik het doen, al ben ik weinig bevoegd. Vergeet daarbij echter in geen geval het bijbelwoord «arste maket yv seluen gesunt ». De bewijsplaats luidt: "gy hebbet van oetmodiger mynnen van my begheert als dat ic yv een luttinck scriuen wolde wo gy yv hebben vnde regeren solden na yuwen state daer gy in gesat syn ouermyts ghehoersamheit yuwes ouersten Meer want ic my daer seer vnnutte ongheleert vnde onbeleuet to kenne yemant to leren so entsee ic my wat to scriuen... ».

d) Gescheiden van de aanleiding lijken mij de volgende passussen die de wil om te schrijven accentueren (dispositio) :

«Meer want ic weder anse yuwe oetmodighe vnde mynlike begherte so en can de mynne nicht wegheren "), "Want ic hoer dat vnse mynlike here seghet do up dynen mont vnde ic sal en vervullen »,

"Vnde hijr vmme wil ic op synen troist to synen loue vnde to syner eren beghinnen vnde $\mathrm{yv}$ een luttinck scriuen also alset de mynlike here geuet ».

(31) Zie G.R. Hocke, Manierismus in der Literatur (Hamburg, 1959), blz. 237. 
II. Tekst

A. Algemeenheden

a) Alle goede werken moeten ter ere van God opgedragen worden. Volg Christus na "in oetmodighen vnde mynliken werken »; wees zachtmoedig en draag geen haat.

b) Beschouw uw overste als Christus en uw medebroeders als de apostelen. Neem $\mathrm{u}$ voor $\mathrm{u}$ te beteren wanneer $\mathrm{u}$ niet in orde was; zwijg wanneer $u$, ook zonder gegronde reden, berispt wordt.

c) Theorie baat niet : er moeten werken zijn. En die werken moeten ook voleind worden. De mens moet bereid zijn «myt rechten herten to allen guden werken te staen $\gg$.

B. Praktijk

d) De kok moet speciaal de zieken helpen; hij zal voor hen alles vriendelijk doen wat de ziekenmeester heeft voorgeschreven. Hij zal zich oefenen in het medelijden.

e) Naast « alle oefninghe de ghy hebben mochet in lesen of in ghebeden » moet de kok werken in minne en gehoorzaamheid. Bescheiden werk, in een geest van gehoorzaamheid volbracht, weegt op tegen aanwezigheid in de kerk: "gy moghet meer myt ghehoersamheit verdenen in der koeken dan myt eyghenen willen in der kerken $\gg$.

f) Sta 's morgens vlug op en bid; dank God intens «myt inwendighe begherte »: liever slechts één salm goed bidden dan in vlug tempo een heel salter. Oefen u dan in de gehoorzaamheid vanaf het ogenblik waarop u « of clemmet van yuwer camer»

- «als gy vuer maken sollet vmme yuwe spise to reden »,

- «als gy to den putte ghaet vmme water to putten»,

- «als gy de vate nemet daer gy de spise in reden sollet»,

- «wanneer dat gy de potte roret »,

- «wanneer gy de spise schumet»,

- «wanner gy merket vnde anseet de torue vnde dat holt bernen in den vure $\gg$,

- «als gy de spise vp richten sollet »,

- «als gy de potte vnde schottelen wasschet »,

- «wanneer dat gy beesten of vische dodet of ter kocken houwet vnde bereyt »,

— «als gy to mysse gaet»,

- «wanneer gy hoert dat yemant cloppet $\mathrm{v} m \mathrm{me}$ yv to hebben of to spreken 》, 
- bid 's avonds niet zo lang als ge moe zijt, maar doe het dan « myt corten ghebeden ».

Al deze praktische raadgevingen heb ik geschreven «al syn se plump vnde grof $》$, maar men moet inderdaad steeds kijken naar Jezus' leven en zijn best doen in alle dingen.

III. Slot

Laten wij dan samen bidden om bescheiden werk, « dit clene », goed te volbrengen.

IV. Index van de kapittels.

V. Datering van het stuk.

\section{SCHRIJVER EN WERK}

De anonieme schrijver moet een geestelijke zijn, waarschijnlijk zelfs een monnik, die de activiteit en de mentaliteit van de kok zeer goed kent.

Boven werd reeds gewezen op de breedvoerige analyse van het kokswerk; in de tekst zelf zitten daarenboven nog ettelijke allusies op het bedrijf in de keuken, zo bv. «al in den brypot roren» ( $7 \mathrm{v})$; «dy altyd pap vnde soite melk geuen; harde corsten eten » $(14 \mathrm{r})$, enz. Dit past heel natuurlijk bij het onderwerp: raadgevingen voor een kok.

Vermits in het werk het accent zo gelegd wordt op de innigheid van het gebed - tegenover uiterlijk vertoon - mag men wel denken aan een soort legitimatie van de functie: het werk in de keuken (lees natuurlijk ook: handenarbeid), in gehoorzaamheid volbracht, heeft in Gods ogen meer waarde dan andere geleerde activiteiten.

Het exempel van de twee zusters dat schrijver aanhaalt, en dat gedeeltelijk doet denken aan Maria en Martha, zou kunnen samengevat worden als volgt : caritatieve liefde haalt het op de contemplatieve. Dit is dan de perikope die in omgekeerde richting aangewend wordt. In het bijbels verhaal ligt het accent bij de beschouwende liefde! Hier wordt voorgehouden dat gehoorzaamheid, innigheid, opoffering - in materiële dingen - positieve waarde hebben.

Mocht men het exempel in het ruimer kader van de $15 \mathrm{e}$ eeuw plaatsen, zou men kunnen denken aan sociale factoren als de 
groeiende invloed van de burgerij of aan de opvatting van het werk bij de Broeders van het Gemene Leven.

De kok moet begrijpend omgaan met zieken : hun maag kan niet alles verdragen! Juist door bijdegronds werk golden de koks als weinig teergevoelig of verfijnd; ze moeten aangemaand worden om hun zieken vriendelijk te behandelen.

De gehoorzaamheid tegenover de oversten wordt ook elders besproken vermits zij regel is in iedere kloostergemeenschap. Men zou hierbij kunnen denken aan Jan van Leeuwen : "Siet, dit settic vriwillighe ghehoersamheit in ordenen : dat een monic of een nonne altoes heeft liever te doen ende oec te latene in gherechter bescheidenheit dat haer overste wil ende begheert ${ }^{32}$. Wat de overste beveelt « dat suldi altemale gheerne doen ende laten in gherechter, oetmoedigher, goedertierliker ghedoechsamheit ${ }^{33}$.

Niet zonder reden wordt Van Leeuwen geciteerd. In onze tekst is sprake van « rusebrokes koc de nochtan vijf ammete hadde to verwaren dat was de koeken de ghaste. backen brouwen vnde dat he dat holt moste halen wt den bussche ». Zijn verdiensten zijn groot al was hij «als he seluen seghet vnde ock de ghene tughen diet weten een grof mensche van buten vnde een puer leye ». Onze tekst getuigt trouwens dat zijn inwendig leven sterker was dan dat van honderd monniken of klerken!

De allusie op « rusebrokes koc 》 wijst op bekendheid met een Zuidnederlands auteur, - maar de schrijver verwierf de kennis misschien indirect.

In hetzelfde verband is er nog dit : de auteur deelt een exempel mee $(12 \mathrm{v}-15 \mathrm{r})$ dat zich afspeelt «by eyner stat de hed sunte truden vp aspeghouwen in den stichte van ludick», - dit is in het werk de tweede expliciete verwijzing naar het zuiden. Het exempel werd gekozen omdat één van de beide zusters "plach de koeken to verwaren » (13 r).

De schrijver grijpt naar eenvoudige, maar plastische beelden. Er werd reeds gewezen op het feit dat iedere broeder, bij de overweging, die middelen gebruikte die best pasten bij zijn verstandelijke ontwikkeling. Zo bv. liet een kok, Helmicus, op de muren van zijn cel in het Scholierenhuis te Zwolle een duivel schilderen en trachtte hij om «ter wille van de gedachte aan den naderenden dood, van de huid van een opgeknoopten misdadiger voor zich een hemd laten maken ${ }^{34}$. Zo wordt ook hier de kok bv. herinnerd

(32) J.W.N. Delteyk, Jan van Leeuwen en zijn tractaat: Van vijf manieren broederliker minnen (Utrecht-Nijmegen, 1947), blz. 134.

(33) Ibid., blz. 135 .

(34) St. Axters, op. cit. III, blz. 70-71. 
aan het feit dat de vissen die op tafel komen, moeten «betalen dat se gheten hebben ». De toepassing luidt dan: de mens die «na den wtwendighe synnen als een beest » geleefd heeft, zal dan ook « van den helschen kocken... to den ewighen vure ghebracht weerden daer se ghebraden vide gesoden sollen weerden $\gg(35 \mathrm{r})^{35}$.

De eenvoud van de tekst blijkt ook uit het feit dat de typische woorden, die behoren tot de technische woordenschat van de vroomheid, weggelaten werden. Zo komen niet voor, in figuurlijke betekenis, termen als : dorsten, drincken, eten, spisen, hongher en dgl. die toch tot de sfeer van kok en keuken behoren en die hier gemakkelijk konden aangebracht worden, naar het voorbeeld van de mystieke taal.

Latijnse citaten of teksten ontbreken.

Men kan zich best een idee vormen van het verschil tussen een echte mystieke tekst en ons hs. indien men bv. het devoet epistel vergelijkt met een Tauler. In beide teksten worden identieke bijbelcitaten aangehaald, maar de illustratie is totaal anders. Men vgl. de eenvoudige verklaring bij «So we na my wil comen de boer vp syn cruce vnde volge my na » (2 v) met de lange en veel moeilijker uiteenzetting bij J. Tauler (uitg. A.L. Corin, dl. II [LuikParijs, 1928], blz. 301 vv.). Ofwel de «oefening » op het eind met Tauler (ib., blz. 239-255), een tekst die Corin noemt «Un abrégé des pratiques spirituelles ».

De titelaanduiding 《in eenen reguler closter 》 kan wijzen op een klooster van de Augustijnen; deze orde was in Westfalen en in de Nederlanden meestal aangesloten bij de Windesheimer kongregatie. Indien deze voorstelling juist is, dan werd het devoot epistel geschreven in de kring van de Broeders des Gemenen Levens.

De grenssituatie of de kontakten van deze gemeenschappen over de grenzen heen stelt men vast in het vokalisme en in de woordenschat.

Oostelijk zijn vormen zoals een luttinck, vnsen, nicht (= niet), bodeschop, reeschop (= gereedschap), leue broder en dgl., misschien ook rekelic, een woord dat in deze vorm slechts in het Nederrijns werd opgetekend, maar «misschien is hij ook in het Oostmnl. bekend geweest », naast het gewoon reckelic (zie Mnl. Wdb. 6, 1246).

Verwarring tussen oostelijke vormen en oostelijk Nederlands is er in: gy scbicket naast gy zweten; dat gy daer vp mercken vnde

(35) Vgl. de kokende ketel in de hel waarin, blijkens de Sermoenen op de tien geboden, een man en een vrouw werden gegooid "ende sy soden ende walden oec in die cupe up ende neder ghelijc eerweten in de potte pleghen te sieden", apud C.G.N. de Vooys, Middelnederlandsche Legenden en Exempelen ('s-Gravenhage, 1900), blz. 292 . 
dencket; dat wij beghinnen... sult en wij solle of sollen.

$G y$ en $d u$ staan door mekaar : dat sulle gy nemen; so en solle gy nicht arguweren; gy sult; gy sollet; du salt, so saltu.

$O L$ is gewoon voor het westelijk $O U$ : old, solde, wolde, bolden, manichuoldicheit, enz.

Sommels, vergadderen (= vergaderen) en dgl., net zoals wo, woe zijn oostmnl. Het laatste is zelfs een typisch compromis tussen het oostelijk wie en het westelijk boe.

\section{BEWIJSPLAATSEN}

Er zijn heel wat bewijsplaatsen die meegedeeld worden met hun bron, zo bv. "Hugho van sunte victoer seghet» (16 v), "Sunte Gregorius seghet » (21 r), enz. Maar door de band kunnen de bewijsplaatsen teruggebracht worden tot twee grote groepen; bijbelse teksten en de Vitae Patrum.

De bijbelteksten worden meestal ingeleid door : « dat ghescreuen staet », «dat onse mynlike here seghet », «na den woerden vnses mynliken heren » en dgl. Voorbeelden :

" de seggen sal wat gy eynen van dussen mynnesten ghedaen hebbet dat hebbe gy my gedaen (2 r) (vgl. Matth. 25, 40);

" de ock seghet Ic en byn nicht ghecomen dat men my dene meer dat ic dene (2 r) (vgl. Matth. 20, 28; Marc. 10, 45; Luc. 22, 28);

" de seghet So we na my wil comen, de boer vp syn cruce vnde volge my na » (2 v) (vgl. Matth. 10, 38; 16, 24; Luc. 9, 23; 14, 27; Marc. 8, 34; 10, 21);

" yuwen schat vergadderen in den hemel daer he yu van nemant ghenomen en mach weerden » (2 v) (Vgl. Matth. 6, 19-21; Luc. $12,33-34)$;

«sachtmodich to wesen als eyn lam... » (4v) (vgl. Isaias 23, 7); «Selich syn de vredesam. of vredemakers... » (4v) (vgl. Matth. $5,9)$;

« en laet yv nicht verdreten een ezel vnses leuen heren to wesen. vnde merket dat de prophete to vnsen heren seghet in den salter Als eyn beest bin ic ghewoerden (6 v) (vgl. Ps. 73, 22);

« dat gescreuen staet in den salter he heuet ghestroyet ende ghegeuen den armen syn rechtuerdicheit 》 (8 r) (vgl. Ps. 112, 9);

«dat sunte Jacob de apostel seghet Nicht de de ee horen of lesen meer de se vulbrenghen mytten werken de sullen gherechuerdichet weerden » (8 r) (vgl. Jac. 1, 22, 25; 2, 17-18), enz.

De Vitae Patrum ingeleid als : "wy lesen in der vader leuen» of «als men leset in der vader leuen » zijn, in de tekst, excerpten 
uit een kompilatie van het zgn. Vaderboec en van andere werken die het leven van de vroegste monniken beschrijven (zie C.G.N. de Vooys, op. cit., blz. 13-14).

De tekstuitgave die hier volgt, is volledig diplomatisch; de afkortingen werden in cursief weergegeven.

Hir beghint een deuoet epistel ghescreuen enen $k o c$ in eenen reguler closter ${ }^{1}$

$\mathrm{DE}^{2}$ vrede godes de alle synnen bouen ghaet de mod behoden vnde bewaren iv herte vnde yuwe synne vnde mote yuwe verstandicheit verclaren to synen loue vnde to syner eeren in vnsen leuen heren ihm xpm Leue broder in vnsen leuen heren gy hebbet van oetmodiger ${ }^{3}$. mijnnen van my begheert als dat ic yv een luttinck scriuen wolde wo gy gv hebben vade regeren solden ${ }^{4}$ na yuwen state daer gy in gesat syn ouermyts ghehoersamheit yuwes ouersten Meer want ic my daer seer vnnutte ongheleert vnde onbeleuet. to kenne yemant to leren so entsee ic my wat to scriuen vnde merke dat ghescreuen staet medice dat

(1v) is arste maket yv seluen gesunt Meer want ic weder anse yuwe oetmodighe vnde mynlike begherte so en can de mynne nicht wegheren Want ic hoer dat vnse mynlike here seghet do up dynen mont vnde ic sal en vervullen $W$ ant he is de gene daer alle gude werken in beghinnen vnde enden Vnde hijr vmme wil ic op synen troist to synen loue vnde to syner eren beghinnen vnde yv een luttinck scriuen also alset de mynlike here geuet vp dat syn soite name daer in gheeert ${ }^{5}$ vnde ghelouet weerde Vnde ysset dat gy dyc luttike to werke settet ic hope yv sal meer ghegeuen weerden van vnsen mynliken heren. Woe een goetwillich koc inden erster sinen staet anmerken sal unde bem pinen den te ordineren totter mester eren godes. Dat ierste capittel ${ }^{6}$.

(2r) In den eirsten suldi leue broder merken dat ghescreuen staet na des apostels lere dat een yegelic mensche em sal pynen weerdelike vnde redelike sal wanderen in synen staet daer he van gode in gheropen is also dat he gode behage Vnde want vnse mynlike here yu gheropen heuet in enen wtwendighen guden leuen vp dat gy em denen sollet in synen leden de seggen sal Wat gy eynen van dussen mynnesten ghedaen hebbet dat hebbe gy my gedaen Vnde vp dat gy em volget de ock seghet Ic en byn nicht ghecomen dat men my dene. meer dat ic dene vnde myne sele sette vor alle menschen $\mathrm{Hijr}$

(1) Titel volledig rood.

(2) De eerste majuskel is rood.

(3) De -e-van oet- is bovengeschreven.

(4) $\mathrm{De}-l$ - is later bovengeschreven.

(5) De derde -e- werd later bovengeschreven.

(6) De kursief gedrukte tekst is rood. 
$\mathrm{v} m$ me leue broder is dat gy yv hijr to geuen wilt So solle gy myt groten ernste merken dat gued is vnde wat vnse mynlike here

(2v) van $\mathrm{yv}$ begheert Dat is dat $\mathrm{gy} \mathrm{yv}$ seluen allene ordelen sollet vnde waerachtelike yuwe sunde vnde ghebreke. bekennen vnde dat gy leifhebben solt barmherticheit vnde guderterenheit hillichlike vnde doeghensamlike myt yuwen broderen to wanderen. vnde dat ghy sorchuoldich vade ernsttichtich. syn vnsen leuen heren na to volgen de seghet So we na my wil comen de boer vp syn cruce vnde volge my na Dat is in oetmodighen ${ }^{7}$ vnde mynliken werken Vnde dat alle tijd to synen loue vnde to syner ere also dat he beghin vnde ende sy al yuwer guden werke. vnde so solle gy gude vrucht voertbrenghen ${ }^{8}$ vnde yuwen schat vergadderen in den hemel daer he yv van nemant ghenomen en mach weerden Want na den woerden ${ }^{9}$ vnses mynliken heren Alle plantinge

(3r) de syn hemelsche vader nicht gheplantet en heuet de sal wtgherodet weerden Dat is alle de gude werke de to synen loue vnde in em nicht ghedaen vnde beghunnen en syn de en sollen nicht verdenscht ${ }^{10}$ wesen to den ewighen leuen Vnde hijr vmme is vns sunderlinge noet dat wij in al vnsen guden werken vns pynen eyne rechte vnde gude meninghe to hebben Want vnse mynlike here ordelt. meer na der meninghe dan na den werken van buten Vnde aldaer solle wij vns pynen dat et eynde vnde dat beghin al vnser guder werke of arbeydes god sy vnde dat wij lof vnde eere daer sympelike ynne soiken sunder enighe twiuoldicheit vnde so en koene wij nicht bedroghen weerden Gy solt yv pynen al yuwe werke myt deuoter

(3v) herten to done betruwende vp de guetheit godes. dat he seluen yuwe loen sal wesen want van den mynsten werke of arbeyt dat wij doen. of laten to den loue vnde to der ere godes daer solle wij vnsprekelike vrucht vnde loen af crighen vnde entfangen ${ }^{11}$ in den tocomende leuen de ewelike bliuen sal vnde nicht verghaen Als wij lesen in der vader leuen dat de hilligen vaders een stro plaghen wt der kerken to dreghen. vnde meenden daer groit loen af to krighen. Hijr vmme leue broder solle gy yv pynen myt vurighen begheerten to arbeyden. in allen guden werken de wile dat gy tijd hebbet als vnse leiflike here seghet Wandert de wile dat gy lecht hebbet. vp dat yv de dusternisse. nicht en begripe. want wo langhe dat gy leuen solt dat en

(4r) ghenedich ontfarmych medelidende mylde van dat gy hebbet na beschedenheit. guderteren to sprekelic eynen ytliken de yuwer behouet Gy en sult nemant haten noch benyden versmaen noch bedrucken myt harden of versmaliken woerden. meer alle dinck myt

(7) De -e- van oet- werd bovengeschreven.

(8) Idem voor de -e- in voert-.

(9) Idem voor de $-e$ - in woerd-.

(10) Het w. werd in het Mnl. Wdb. niet opgetekend. Het Mnd. Wtb. van Schiller-Lübben V, 334 geeft 'vordensten' = Dienste von einer Sache leisten.

(11) De -t- in ent- werd bovengeschreven. 
sachtmodighen herten leren draghen. vnde vergheuen so we teghen yv doet in woerden of in werken Nemant en solle gy bespotten noch onweerdicheit ${ }^{12}$ beden in woerden noch in werken myt tekenen noch myt ghelate in ghener wijs $G y$ en sult nicht wesen stuer noch zuer. meer soetelic rijp van seden vnde blide van angesichte. meer nicht lichtuerdich van seden noch van gelate Gy en sult vp nemant mystruwen noch nemant ordelen van den dinghen de yv verborghen syn $G y$ sult $\mathrm{yv}^{13}$

(4v) pynen sachtmodich to wesen als eyn lam dat men to den dode ledet dat nochtan nicht toernich ${ }^{14}$ en wert Leert duken swyghen vode liden vnde latet ouer $\mathrm{yv}$ ghaen so moghe gy alle tijd vrede van herten holden vnde so hebbe gy alle tijd den besten coep Vnde aldus moghe gy vercrighen daer vnse mynlike here van seghet Salich syn de vredesam. of vredemakers want se sullen kinder godes gheheten weerden

Woe be bem bebben sal inder teghenwordicheit sines ouersten vnde sine broederen dat derde capittel 15

GI ${ }^{16}$ sult yuwen ouersten anseen. als vnsen leuen heren nicht als eynen menschen. vnde yv pynen sunderlinghe in syner yeghenwordicheit yv oetmodelike vnde ersamelike to hebben nicht koen-

(5r) like. noch lichtuerdelike De woerde ${ }^{17}$ de he $\mathrm{yv}$ seghet of dat he yv beuelt dat sulle gy nemen van den monde vnses leuen heren de van em vide van syns gheliken spreket vnde seghet. de yv hoert de hoert my de yv versmaet de versmaet my. wanneer. gy van em of van den procurator. berispet wert so en solle gy nicht arguweren of yv entschuldighen. $\mathrm{vp}$ de tijd meer gy sult oetmodelike neder uallen vnde seggen dat $\mathrm{gy} \mathrm{yv}$ beteren wilt vnde menen ock dat mytten herten isset dat gy schuldich syn Isset dat gy vnschuldich syn so moghe gy em na allene de sake of waer $v m m e$ dat schach segghen myt sachtmodigher oetmodicheit isset noet to segghen isset ghene sunderlinghe noet so solle gy swighen vnde liden

(5v) vnde nemen schult daer gy gheen en hebben so moghe gy vnsen mynliken. heren ihu xpo wat gheliken de vnse sunde vnde vnse schult betaelt. heuet myt synen bitteren dode vnschuldichlic vp dat he vns salich maken mochte De procurator dem de dinghe van buten beuolen syn de solle gy hebben als sunte peter den vnse leue here syne hilligen kerken vade syne schape beuolen heft De brodere solle gy anseen als de hilligen apostelen vns leuen heren of als de engele godes $\mathrm{Al}$ de anderen broders ${ }^{18}$ leyken vnde ander solle gy anseen als de hilligen dyscypulen vns leuen heren of syne vercoren sone den he syn rike bereyt heuet Vnde aldus solle gy de

(12) De tweede -e-in onweerd- werd bovengeschreven.

(13) Onderaan de kolom, in jonger schrift: He (voor Here?) Jobannes.

(14) De -e- in toern- is bovengeschreven.

(15) De gekursiveerde titel is rood.

(16) De eerste majuskel is rood.

(17) De -e- in woerd- is bovengeschreven.

(18) Met bovengeschreven - 0 - 
dynghen myt merer deuocien vnde mynnen doen als gy

(6r) leuen heren alre naest was $\mathrm{Al}$ was dat he nicht soitelike synghen en conde nochtan he was mede in der scharen mytten apostolen vnde mytten anderen de lude songhen vnde de apostel. legheden er cleder vp em vnde somighe spredden or cleder in den wech vp dat he daer vp ghaen solde vnde breken toeghe van den palmen vnde streygeden se vnder syne voite in den weghe vp dat he daer vp treden solde vnde ouerghaen. vnde de ezel quam also wal to Jerusalem als de anderen deden de lude songhen. Meer nochtan solle gy merken. al isset dat de brodere schinen van buten dat se it beter hebben dan de leyken. vnde de to arbeyde geset syn. vnde daer vmme sommels segghen. In wat hebben de monike to noede se hebben oer butscho ${ }^{19}$ an vnde hebbe se to luttinck an eynen

(6v) pelse se trecken twe an Leue broder dat en leghet in den pelsen van buten nicht. meer se hebben last van bynnen vnde van buten. want se moten ock wo de feste merer syn wo se merer arbeides hebben van buten. vnde moten nochtan liden manich druck vnde teghenheit de yv vnkundich syn. Vnde dat weyt ic wal solden de ghene de dat seggen em volghen nacht vnde dach in eren arbeyet van bynnen vnde van buten al wolde ic em dre pelsen an trecken vnde twe paer butscho dat solde em nochtan verdreten. want se en weten nicht wat se segghen Vnde hijr vmme mijn leue broder en laet yv nicht verdreten een ezel vnses leuen heren to wesen. vnde merket dat de prophete to vnsen heren seghet in den salter Als eyn beest byn ic ghewoerden ${ }^{20}$

(7r) by dy. vnde ic byn altijt myt dy Vnde isset dat gy aldus yv schicket vnde yw veroetmodicht so sollen de apostolen dat syn al de broders er cleders dat syn oer doegheden vnde gude werke vp yuwen rugge leggen vnde yuwe crancheit. vnde onvulcomenheit daer mede bedecken als ghescreuen staet Salich syn de ghene de er sunde bedecket syn want dat $\mathrm{yv}$ ontbreket in rechter vulcomenheit dat sollen se vervullen want de mynne bedecket manichuoldicheit. der sunde Vnde hijr vmme want gy myt mynnen bedecket syn so sal god vnse here de de mynne is vp yv sitten ${ }^{21}$ vnde yv regeren vnde sturen in allen goden werken vnde in den rechten wech Vnde want gy vermyts menijchuoldicheit der vnlede altijd nicht merken en cont waer gy yuwe voite der begherten setten sult of

(7v) waer de wech sachte of hart is hier vmme sullen yuwe brodere dat de kinder godes syn oer cleder vnde telghen vnde den palmen in den wech stroyen. dat is al oer vurighe meninghe vnde vpsate der doeghede vnde al dat se lesen syngen of van buten arbeyden of collacien of in den lesen der hilligen scrift vercrighen. dat sullen se yw vermyts mynne. vnder spreyden op dat gy sachte vnde seker ghaen moghet vnde dit sal ghehoersamheit al vulbrenghen vnde

(19) Oudemans I, 774 en Mnl. Wdb. I, 1393 geven hetzelfde citaat ; de eerste vertaalt slechts door 'laars', het Mnl. Wdb. door 'een soort grove schoenen'.

(20) De tweede $-e$ - is bovengeschreven.

(21) De kopiist schreef eerst syt-. 
de mynne sal dat alghemene maken Want al dat de ander doen de in den cloester syn van ynnighen vnde guden werken dat solle gy al in den brypot roren is dat gy de doeghet in eynen ytliken broder ${ }^{22}$ mynnet. vnde dan solle gy dat weder wtdelen ouermyts der seluen mynnen alle den ghenen de es behouen vnde

(8r) van yv begheren Vnde aldus sulle gy dat vulbrenghen dat gescreuen staet in den salter he heuet ghestroyet vnde ghegeuen den armen syn rechtuerdicheit. sal bliuen in ewicheit der ewicheit Vnde aldus moghe gy ock vervullen dat sunte Jacob de apostel ${ }^{23}$ seghet Nicht de de ee horen of lesen meer de se vulbrenghen mytten werken de sullen gherechuerdighet weerden Vnde hijr vmme al isset dat de mensche vele leset wo he doen sal vnde dat weet vnde nicht to werke en set als he schuldich is to done so en batet em syn lesen noch syn weten nicht meer et is em meer verdomenisse wat daer staet ghescreuen. de knecht de daer weet den willen des heren vnde nicht en doet de sal myt vele wonden ghequesset of gheslaghen weerden Vnde hijr vmme solle gy yv

(8v) pinen to hebben luttinck woerde vnde vele leuens vade pynen $\mathrm{gv}$ alle tijt to stane vp ghehoersamheit yuwes ouersten so en conne gy nicht bedroghen weerden. want he zwemmet. of driuet sachte wes kin van eynen anderen vpgholden wert Vnde hijr vmme weset vnverveert al isset dat yv dusse of ander becoringhe to comet. meer doet als de prophete seghet verbeide den heren vnde werke manlike vnde dijn herte sal ghesterket weerden Vnde ock als sunte iacob de apostel seghet Selich is de man de becoringhe lidet want als he gheprouet is so sal he entfanghen de crone des leuens de god belouet heft den ghenen de ene leifhebben

(9r) Daer ${ }^{24}$ staet gescreuen in den olden testamente dat vnse leuen here gheboet dat men van allen offerhande solde offeren houet vnde steert Daer is vns by betekent dat wij nicht allene gude werke beghinnen en sult mer daer by bliuen to den ende Vnde hijr af heuet vns vnse mynlike here seluen exempel ghegeuen want do de ioden segheden bistu conninck van israel ganck van den cruce vnde wij sollen dy ghelouen he en wolde des nicht doen. noch vns exempel geuen de vnvolstandicheit. meer he bleif an den cruce vnde starf an den cruce Vnde he waert van rechtuerdighen menschen af ghedaen vp dat wij nicht en mochten seggen Wat solde ic an den cruce bliuen. want vnse here genck seluen vanden cruce Meer vp dat wij

(9v) an den cruce solden bliuen vnde em na uolghen vnde ghelijc als he van guden menschen af ghenomen waert Also sollen vns de hilligen engel af nemen vnde voren vns daer wij eweliken rasten sollen isset dat wij hijr in vnsen arbeide vmme godes willen vulstandich bliuen vade vns van gheenre becoringhen laten verwinnen

(22) $\mathrm{De}-0$ - is bovengeschreven.

(23) $\mathrm{De}-l$ - is bovengeschreven.

(24) Met rode majuskel. 
Als ghescreuen staet Isset dat enich gheest de macht ${ }^{25}$ heuet vp di comet eyn wille dine stede nicht laten Dat is worstu van bynnen of van buten becoert dijn gude vpsaet of beghin to laten wes em nicht ghehoersam meer hoer ${ }^{26}$ wat ghescreuen staet So we vulstandich. bliuet to in den ende de sal gesunt weerden Vnde na den woerden ${ }^{27}$ sunte pawels Also solle wij lopen dat wij gripen daer wij

(10r) na lopen. Anders lope wij to vergeues is dat wij ynt myddel of by den ende bliuen staende Vnde hijr vmme laet yv nicht verdreten eyn luttinck arbeydes to done vmme de mynne vnses leuen heren want gy sollet noch segghen Ic hebbe luttinck ghearbeidet. vnde ic hebbe grote raste ghevunden Want al de dropen zwetes de gy zweten in den arbeide vmme vnses mynliken heren willen vnde al de voetstappen de gy ghaet de weerden al van yuwen engel ghetellet. Als men leset in der vader leuen van eynen broder de plach sijn water een lanck stucke weghes to halen ten lesten begant em to verdreten. vnde dachte he wolde eyne putte neger maken vp dat he nicht so veer gaen en dorfte. Do he aldus dachte. do hoerde he eynen achter

(10v) em komen Do sach he vmme vnde vraghede we he weer. Do seghede hi Ic byn dijn engel vnde ic volge di na vnde telle alle de voetstappen de du ghaest want van elleken saltu loen entfangen do he dat hoerde do makede he synen putte noch eyns also veer als he to voren stont vp dat he vele loens entfangen. mochte Vnde ock lest men van sunte beerndes broeders dat vp een tijd do se arbeyden in den bouwe vnde dat se seer bezwaert vnde bezweet ${ }^{28}$ weren do sach eyn hillich man dat vnse mynlike zoite vrouwe de hillige yuncfrouwe maria quam myt eynen schonen doke vnde droghede de brodere van den swete Hijr vmme solle gy exempel. nemen van eyner hilligen vrouwen de plach vele to arbeyden vnde

(11r) ghaen in den denste godes vnde se hadde eyn quaet ${ }^{2 \theta}$ been dat plach er to male seer to done als se also moede arbeyde Alset oer dan wee dede vnde se wat doen solde of ghaen. so sprack se to eren bene vade seghede leue been ghanck nv voert vnde help my dat ic dat werck der mynne ${ }^{30}$ veruullen. mach so saltu noch so claer vnde so schone weerden als de sunne vnde du en salt nimmer meer pine liden vnde aldus genck se vaste voert vnde troste er been vnde arbeide to den dode to. vnde do vercrech se daer se vmme arbeide Vnde aldus solle gy ock doen vnde troisten yv seluen als gy beswaert weerden want et en sal nicht lange dueren Hijr vimme wandert vnde arbeidet de wile dat gy tijd hebbet vnde de wile dat it sommer is vp dat gy in den wynter

(11v) dat is als gy steruen sult of als gy nicht meer arbeiden moghet

(25) Verbeterd uit nacht.

(26) mer bor met bovengeschreven -e-.

(27) Met bovengeschreven $-B$ -

(28) Boven de $-t$ - staat een andere $-t$ -

(29) quaet werd een eerste maal doorgehaald.

(30) der mynne werd in de rand rood bijgeschreven. 
rasten moghet Vnde dan solle gy horen dat de prophete seghet wantu den arbeit dyner hander eten salt salich bistu vnde dy sal wal ghescheen

Wo dat be nicht beswart en sal wesen als be bem dorre unde vnbegberlic ghenoelet unde wat waer deuocie is dat vj capittel ${ }^{\mathbf{a 1}}$. Want ${ }^{32}$ de ghene de in manichuoldicheit becommert syn vnde in wtwendighen vnleden dickewyle duncket dat se dorre vnde sunder deuocien syn so weerden se somtijd bezweert vnde em twiuelt vnde hebben anxt of er arbeit vnsen leuen heren behaghelic sy of nicht want se gheen soitecheit noch deuocien. in eren ghebede en volen daer of leue broder solle gy weten. dat dat nicht allene ynnicheit of

(12r) deuocie en is dat een mensche vele leset of synget of soiticheit voelt van bynnen in synen ghebede of dat men scryet. Dit hebben alte male wal sommetyd wertlike menschen. de nochtan na den vleische leuen Want we hijr vp slichtes rastet vnde pynet em nicht to werken als daer to hoert ${ }^{83}$ de wort bedroghen Meer gy sult weten dat dit rechte deuocie is na der lere der hilligen Dat eyn mensche bereyt sy myt rechten herten to allen guden werken daer de ere godes vnde mynne des naesten ynne ghelegen is vnde dat eyn mensche seluen sy een offerhande. vns heren. Also dat he myt vurigher meninghe em seluen late vnde ouergheue in den handen vns leuen heren vnde syns ouersten $\mathrm{V}$ nde dat he alle dinghe de vp em vallen

(12v) van buten of van bynnen neme van der hant godes vnde em alle tijd danckbaer sy Als de prophete seghet Ic sal benedien den heren in aller tijd dat is in voerspode ${ }^{34}$ vnde in teghenheit vnde dit is rechte deuocie Vnde hijr af wil ic yv een exempel scryuen Dat weren twe suster de beyde van guden willen waren vnde hadden em afghescheden van der ghemeynheit der werlt by eyner stat de hed sunte truden vp aspeghouwen in den stichte van ludick Dusse twe suster wonden to samen De eyne was seer inwart ghekeert vnde gaf er seluen to male ouermyts de gracie godes to vnsen leuen heren Also dat se daer to quam dat vnse guderterene here em er dickewile plach to openbaren als eyn kindeken vnde anders manigher

(13r) hande wijs vnde plach oer dickewile solaes vnde ghenoichte to done vnde myt oer to spreken De ander suster plach de koeken to verwaren vade de ander dinghe van buten vp dat oer suster also vele de bet vnsen leuen heren denen mochte vnde oer to inwendicheit vnde to ${ }^{35}$ deuocien. gheuen vnde se plach luttinck of nicht to volen van sulken inwendighen. dinghen meer se was altijt in zwaerheit vnde dorricheit des herten Vnde want er suluen mysherghede dat se also vngheneecth ${ }^{36}$ was vnde dorre was so bad se vp eyne tijd

(31) Gekursiveerde titel rood.

(32) Met rode majuskel.

(33) Met bovengeschreven -e-

(34) -po- werd verbeterd uit een niet meer te ontcijferen verschrijving.

(35) $\mathrm{Na}$ to staat een doorgehaald begin devi.

(36) Wel een verschrijving voor vngheneecht, d.i. mnl. ongeneiget. 
er suster vnde seghede Och leue suster wolde gy mijner ghedencken als vnse leue here by yv comet vnde vor my bidden vnde em vraghen of ic ock in synen oghen bekant sy. vnde of he myner icht achtet want ic vruchte dat em

(13v) myn leuen vnde myne werke nicht en behaghen vmme des willen dat ic also dorre vnde sunder begherte byn vnde altijt in wtwendighen dinghen vnledich byn Do louede oer er suster dat se de bodeschop doen wolde als se mende dat it tijd vnde nutte weer ${ }^{37}$ Hijr na nicht langhe so quam vnse leue here als he ghewoentlic was to der suster. do se to samen in ghenoechten weren vade der suster duchte dattet tijd was. Do seghede de deuocie suster to vnsen leuen heren $\mathrm{O}$ mynlike guderterene here weert dat ic gracie gheuonden hedde in yuwen oghen vnde wiste dattet yv ghename weere so wolde ic yv oetmodelike. bidden vor myne suster de in wtwendighen werken vnledich. is dat gy erer entfarmen wilt vnde of se yv in eren werken behaget

(14r) want er duncket dat se gheen betruwen. en heuet vp er seluen noch vp er wercke Do antworde oer vnse leue here wat scharpelike also dat de suster al vorsaghet vnde verslaghen waert vnde seghede wat biddestu voer ${ }^{38}$ dijn suster du heuest meer to done dat dijn suster vor di bidde Want dy moet ic al myt smeken holden. vnde dy altijd pap vnde soite melk geuen vnde weert dat ic dat nicht en dede du soldest my entghaen Meer dyner suster gheue ic harde corsten eten druck becoringhen vnde maniger hande zwaerheit. vnde dit nemet se al guderteerlike. van myner hant vnde is my danckbaer in allen vnde aldus hebbe ic se als mijn eyghen beseten vnde mach myt er doen al dat ic wil vnde in dussen is se my seer angheneme. vnde behaghelic Vnde hijr vmme

(14v) bidde oer ${ }^{39}$ dat se vor dy bidde Want du heuest des bed to done dan see Do vel se neder vor de voite oeres ghemynden vnde bad ghenade vnde dat he er cranckheit vnde vnvolcomenheit to hulpe wolde comen. vnde seghede dat se dat gheerne doen wolde Vnde aldus ghinc se to erer suster vnde bad er dat se vor oer bidden wolde vnde se seghede oer alte male als vnse leue here oer gheseget hadde do was de ander suster seer verblidet in vnsen. leuen heren dat he errer ghedachte ${ }^{40}$ vnde seghede Want mijn mynlike here in myner armoden myns ghedechtich. is vnde em myne wercke ghename syn. so en wil ic my voert ${ }^{41}$ an gheen arbeydes laten verdreten meer ic wil em ghetruwe wesen also langhe als ic leue Vnde hijr vmme leue broder weset vulstandich to den

(15r) ende. vnde so solle gy de crone des leuens entfanghen de vnse mynlike here belouet heuet den ghenen de vulherden in der seken

(37) Met bovengeschreven - $e$ -

(38) Met bovengeschreven -e-.

(39) Met bovengeschreven -e.

(40) ghedachte is het laatste woord op de regel; -te-werd klein bovengeschreven om niet te zeer buiten de bladspiegel te geraken.

(41) Met bovengeschreven - $e$ - 
doeghet to den ende $W_{0}$ be bem sal bebben tot ten sieken unde de synre te doen bebben. vij capittel $\$ 2$

Voert $\$ 3$ leue broder want den ghenen de in sulken ammete of denste gheset is als gy vp dusse tijd syn groet ${ }^{44}$ noet is dat he barmhertich. medelidende vnde gheuoechsam. sy den ghenen de syner behouen vade sunderlinghe den seken vnde den crancken de nochtan to reuenter ghaen vnde sunderlinghe dinghe behouen want se allerhande dinghe nicht verdraghen en moghen vermyts hinder de se daer in den licham af voelen ${ }^{45} \mathrm{Hij}^{46} \mathrm{vmme}$ is yv noet dat gy daer vp mercken vnde dencket vnde dat gy dat yv to den herten latet

(15v) ghaen Vnde dat gy yv pynen den seken vnde den crancken vnde voert alle den ghenen de yuwer behouen guderterelike vnde mynlike to denen van den dinghen de gy hebbet vnde yv beuolen syn. Vnde dat myt mynliken. vnde oetmodighen woerden vnde ghelate van buten $\mathrm{vp}$ dat nemant van $\mathrm{yv}$ vermyts yuwen woerden of ghelate bedrucket of myt rechte beswaert en weerde Gy sult yv merkelike hoeden dat gy nemant en ordelt of em de dinghen de he begheert noet syn of nicht Meer gy sult eynen ytliken beuelen syner consciencie vnde synen ouersten of den sekenmester den de seken vnde crancken beuolen syn want eynen itliken is syn last swaer ghenoich de he draghet. Vnde ock en kan nemant eynen anderen also wal voelen als eyn

(16r) ytlick em seluen Vnde ock leue broder en moghe gy nicht verborghen dat gy vp ander menschen mylde van herten barmhertich gudertieren syn eynen itliken de des begheert dat en weer dat yv van yuwen ouersten van somyghen anders beuolen woerde ${ }^{47}$ mer dat sal $y v$ altijd swaer wesen beholden altijd oetmodighe ghehoersamheit Want gy sult yv pynen also vele als in yv is myt mylder barmherticheit vnde mynnen wt to vloyen to allen menschen. vnde sunderlinge. to uywen broders de in yuwen cloester syn vnde sult yv pynen altijd eyn gued reyne herte to hebben sunder suspicie of quaed vermoden vp yemant Meer gy sult altijd myt mynliken vnde medelidenden herten dencken dat se meer behouen dan se begheert vnde dat se em in mani-

(16v) ghen dinghen vmme godes willen liden de em wal noet weren alset ock in der waerheit dickewile gheschuet. Vnde hijr vmme solle gy yv seer pynen de dinghe de yv beuolen syn also to bewaren vnde to done also dat vermyts yuwer versumenisse of unachtsamheit nemant hinder of ghebreck en lide Gy sult de dinge. de gy doen sollet te voren ouer dencken vp dat gy mytter hast nicht belopen en weerdet want als de dinghen vnverseenliken vp den hals comen

(42) Titel volledig rood.

(43) Met bovengeschreven -e.

(44) Idem.

(45) Idem.

(46) Lees Hijr.

(47) Met bovengeschreven $-e$ - 
so wort de nature. dickewile beswaert vnde verstuert Vnde hijr vmme solle gy weten dat Hugho van sunte victoer seghet dat dat nicht allene. gude ghedachten en syn dat men dencket van der passien vnses leuen heren van der hellen ${ }^{48}$

(18r) groit syn gy nv Vnde he antworde oer ${ }^{49}$ also byn ic in yuwen herten vermeert vmme dat gy my leren vmme my. vnde solaes dat gy van my hadden vmme mynen willen leren vnde ghenget denen mynen leden Vnde hijr vmme leue broder en solle gy nicht waenen dat gy enighes gudes in aldus daniger ghelatenheit sullet berouet weerden Want wat gy van mynnen latet vmme guetwillicheit dat wort yv vele edelre. want vnse here spreket So we icht laet vmme my de sal hundert volt weder entfaen Ock staet in der vader boke van enen hilligen vader de quam to enen anderen seer hilligen vnde vernoemden vader vnde vraghede dussen hilligen vader vnde seghede Vader ${ }^{50}$ by vns wonen twe monike de eyne is seer strenge in vasten in waken in ghebede vnde in den

(18v) arbeide syner hande De ander is medelidende vnde barmhertich vnde pynet em waer he mach den seken vnde den crancken broederen to denen vnde em er noettruft to verweruen we menstu dat de meiste sy voer gode Do antworde de hillege vader vnde seghede Al weer dat em de strenge broder hinghe by den dumen so en konde he nochtan nicht myt syner strengicheit nicht ghecomen daer de broder ${ }^{51}$ to ghecomen is de in oetmodigher mynnen den seken denstachtich. vnde behulpelic is Vnde hijr omme leue broder pynet w barmhertich to wesen vp de ghene de yuwer to done hebben want als ghescreuen staet Salich syn de barmhertighen want se sollen barmherticheit entfaen als se des alre meyst to done hebben sollen dat is als se van hijr scheden

(19r) moten Ock seghet de prophete van vnsen heren. dat syne barmherticheit ghaet of is bouen alle syne werke Vnde de barmherticheit sal bouen dat ordel vnde bouen de rechtuerdicheit wesen Meer somighe menschen willen also strenghe wesen dat se de barmherticheit achterrugghe setten. also dat se gheen medeliden myt anderen luden en hebben of vmme dat se es seluen nicht en behouen. vnde menen dickewile vnde seggen dattet cleyn modicheit. of vleyschelicheit sy vnde se willen al dat een ittelic em lide Meer et vallet dickewile dat de ghene de also syn Als em wat to comet seickheit of swaerheit dat se alre qualekest liden conen vnde dat de meer begheren vnde meer sorchuoldich vor em seluen syn dan ander menschen de se gheordelt

(19v) hebben dusse solden merken dat vnse mynlike here den scriben vnde den phariseen to seghede do se syne discipulen berispeden $\mathrm{v} m \mathrm{me}$ dat vnse leue here at myt matheu $m$ vnde myt anderen ghe-

(48) Fol. 17 ontbreekt; er blijft nog slechts een randje over waarop een paar begin- of eindletters staan.

(49) Met bovengeschreven $-e$ -

(50) $\mathrm{Na}$ Vader een v-teken als verwijzing naar het in de rand bijgeschreven by.

(51) Na broder het v-teken als verwijzing naar het in de rand bijgeschreven to. 
menen sunderen Do antworde vnse leue here vnde seghede. Ghaet vnde leert wat dat bedudet Ic wil barmherticheit vnde nicht offerhande Of he segghen wolde Gy wilt al vp yuwe rechtuerdicheit staen vnde de barmherticheit. wt sluten Vnde gy en voelt nicht dat gy sunder myne barmherticheit. nicht salich en moghen weerden myt al yuwer rechtuerdicheit. de gy doen moghen Daer vmme ghaet in yu seluen vnde merket. wat gy behouet so en solle gy nicht lichtelick vp ander menschen onweerdicheit hebben Ock staet

(20r) ghescreuen van den hillighen bisschope sunte nycolaus. dat he was seer mededoghende vnde een guderteren inwendich medeliden hadde myt allen menschen de bedrucket vnde beswaert weren van bynnen of van buten Hijr v $m$ me staet ghescreuen en wille nicht alte rechtuerdich wesen meer als de apostel seghet sal em een mensche em seluen merken vp dat he ock nicht becoert en weerde want wy alle menschen syn vnde dat den enen huden gheschuet mach morghen vp enen anderen comen Want nemant en is versekert. in dussen leuen als ock de apostel seghet de daer staet de see dat he nicht en valle

Woe dat men ${ }^{52}$ nicht altoes veile en wort de scrifte ${ }^{53}$ lesen ende te boren. mer als men werke der mynne dat viij capittel 54

(20v) Sunte gregorius seghet in der omelien van den twen discipulen de ginghen in emaus vp den paessche dach. Do vnse leue here myt em genck vnde he em bedudde dat in der ee. vnde in den propheten van em ghescreuen was. daer seghet gregorius aldus vp Al was it dat de discipulen de ee vnde de scrift hoerden ${ }^{55}$. ya ock van den monde vnses leuen heren. se en weerden nochtan nicht verluchtet noch en bekanden vnsen leuen heren nicht Meer do se de ee vnde de scrift te wercke setten. vnde do se werke der mynnen begonden to werken do woerden ${ }^{56}$ se verluchtet vnde bekanden vnsen leuen heren Vnde hijr vmme leue broder solle gy yu pynen bouen alle oefninghe de gy hebben mochtet in lesen of in ghebeden dat gy vele

(21r) guder werke der mynnen vnde der ghehoersamheit ${ }^{57}$ werken moghen want dat gy noch also vele leset of bedet of horet prediken. is dat gy dat nicht to werke en settet als gy werken moghet so isset al vmme nicht want et syn vele menschen de altijd lesen vnde horen lesen of collacien nochtan. en connen se nummer to den rechten gronde de waerheit. vnde dat is daer vmme want se dat nicht to werke en setten dat se lesen of horen $\mathrm{Vnde}$ hijr vmme spreket ock sunte gregorius vp een ander stede vnde seghet Isset dattu vele weten

(52) Na men staat be hen doorgehaald.

(53) Boven scrifte staat een $\mathrm{F}$; het woord is een verbetering uit scryte.

(54) Titel volledig rood. - Hij moet foutief afgeschreven zijn; in de index (39v) staat: Wo dat men altijd nicht verluchtet en wert de scrift to lesen vnde to horen. meer als men werke der mynnen doet.

(55) boerd- met bovengeschreven -e-.

(56) woerd- met bovengeschreven -e-.

(57) ghebor- met bovengeschreven -e-. 
willest so sette de werke dattu weest vnde dat sal ghenoech ${ }^{58}$ meer ghegeuen weerden Want al werkende cricht men vnderscheet der doeghenden ${ }^{59}$ vnde dat ene gude werck der mynnen verwecket altijd dat ander isset dat et to rechte

(21v) ghedaen weert vnde in rechter meninghe. Vnde ock seghet vnse leue here Nicht allene de ghene de to my segghen Here here en sullen comen ynt rike der hemele. meer de ghene de doen den willen myns vaders de in den hemel is de sullen dat rike besitten Vnde aldus en leghedet nicht an vele to lesen of horen lesen of prediken. meer dat men vele guder werke der mynnen vnde der ghehoersamheit werke de wile dat men werken mach Vnde hijr vmme solle gy yv pynen mynnen vnde ghehoersamheit vor alle oefninghe der doghede to setten Vnde gheuelt dat gy ouermyts onlede belettet woerden ${ }^{60}$ als dat gy ghene mysse horen moghet of to den hilligen sacramente ghaen daer en solle gy yv nicht in bedrouen noch bezwaert 61

(22r) weerden Want de mynne vnde de ghehoersamheit dat vervullen sal Vnde hijr of lest men een exempel ${ }^{62}$ Dat was vp eynen groten dach dat grawe nonnen van ordenen solden ghaen to den hilligen sacramente vnde eyne yuncfrouwe was daer mede de to den sacramente solden ghaen Vnde als se dat doen solde so quam oer abdisse vnde beuael ${ }^{63}$ er to ghaen in de koeken vnde to done dat daer to done was De yuncfrouwe genc to hant daer men er gheboet sunder murmureren vade myt groter ynnicheit doende dat er gheheten was Do alle de yuncfrouwen dat sacrament entfanghen hadden de sprack daer een stemme vnde seghede De yuncfrouwe de daer in de koekene genck heuet alre ghewaerlikes. de vruchte des sacramentes

(22v) enfanghen ouermyts ghehoersamheit ${ }^{64}$. Unde hijr vmme solle gy yv pinen in allen steden vnsen leuen heren vor yuwen oghen to hebben. vnde yuwe kerke altijd myd yv draghen daer gy syn Want als sunte augustinus seghet Gheloue vnde du heuest ene entfanghen Des gheliken en solle gy nicht verstuert of beswaert weerden al weert dat gy vp somyghen hochtiden nicht to vesper of to metten en moghet ghaen als gy ander werke der mynnen vnde der ghehoersamheit 64 to done hebben of daer yemant $\mathrm{v} m$ me ghebreck of hinder solde hebben Want gy moghet meer myt ghehoersamheit ${ }^{64}$ verdenen in der koeken ${ }^{65}$ dan myt eyghenen willen in der kerken Vnde hijr lest men eyn exempel af van sunte berndes broderen Wo

(23r) dat vp eyne hochtijd als vp vnser vrouwen hemeluaert do alle de

(58) Na ghenoech een v-teken als verwijzing naar het in de rand bijgeschreven meer. Dit laatste moet wel ghenoech vervangen.

(59) doghed- met bovengeschreven -e-.

(60) word- met bovengeschreven -e-.

(61) -zwart met bovengeschreven -e-

(62) Een latere hand schreef hiernaast in de rand: Exemplum.

(63) - ual met bovengeschreven $-e$ -

(64) -bor- met bovengeschreven -e-

(65) -kok- met bovengeschreven -e-. 
brodere de buten waren in den wthouen. to den cloester ${ }^{86}$ pleghen to comen $\mathrm{v} m$ me er hochtijd to holden in eren cloester Vnde dat gheuel do alle de brodere vnde leyken to den cloester toeghen dat eynen leyken beuolen was van synen meyster de den wthof verwaerde dat he bliuen solde vnde des nachtes de beeste hoden vp den velde Dat he oetmodelike vnde ghehoersamlike ${ }^{67}$ dede als eme beuolen was Des nachtes do dachte he wo vnrichlike vnde wo deuotelike dat em de broders do hadden de in den cloester waren vnde dachte dattet ock gued ware al were he in den cloester nicht dat he em daer to vnsen leuen heren ghaeue ${ }^{68}$ als he best mochte want god oueral is Do gaf he em

(23v) seluen mytten herten to vnsen leuen heren vnde kneede dickewile neder vnde las aue maria. vnde aldus brochte he de nacht to Do de metten vte was do waert sunte bernhardus gheopenbaert dat vnsen leuen heren vnde vnser soiten vrouwen Bet behaghede vnde ghenameliker weer des broders deuocie de vp den velde de beesten hodde dan al de deuocie de vp de nacht in den cloester ${ }^{69}$ geweest hadde daer nochtan vele hilliger vnde deuoter broders inne waren Des morghens genck sunte bernhardus vnde dede hijr eynen sermoen af Vor al de brodere vnde leycken vnde seghede so em gheopenbaert was Also dat se alle gader to samen monike vnde leycken daer ynne ghestichtet woerden ${ }^{70}$ vnde verwecket to den werken der ghehoersamheit ${ }^{71}$. Vnde hijr vmme is rechte

(24r) ghehoersamheit ${ }^{72}$ van den hilligen vaders merer gherekent dan doden to verwecken of ander myrakel to done Vnde hijr vmme heuet vnse leue here exempel ghegheuen vnde is ghehoersam ghewoerden ${ }^{73}$ synen vader to den dode vnde to den dode des cruces Als he seluen seghet Ic en byn nicht ghecomen. mynen willen to done meer den willen myns vaders de in den hemel is Vnde hijr vmme staet ghescreuen dat ghehoersamheit ${ }^{22}$ beter is dan offerhande want in den olden testamente plach men to offerne vleisch van calueren vnde van schapen vnde des gheliken dat al buten was. meer $\mathrm{nv}$ in der ghehoersamheit 72 offer wij vns selues vleisch nicht allene vnse wtwendighe. vleisch in den arbeide van buten meer ock vnsen eyghenen willen vnde bereytheit to allen guden werken

(24v) to den loue vnde to der ere godes also dat wij wesen moghen een leuendich instrument godes daer he mede wercken vnde doen mach al dat he wil hijr vnde in der ewicheit Nu hebbe ic vaste ghescreuen wo dat gy yv regeren vnde hebben sult to yuwen mede broders in barmherticheit. in medeliden vode in mynnen $\mathrm{Nu}$ weert

(66) clost. met bovengeschreven -

(67) ghehor- met bovengeschreven -e-

(68) ghaue met bovengeschreven $-e-$.

(69) de - $r-$ is bovengeschreven.

(70) word- met bovengeschreven $-\ell$ -

(71) -hor- met bovengeschreven - $e$ -

(72) -hor- met bovengeschreven - $e$ -

(73) -word-met bovengeschreven -e- 
ock gued dat gy als ghescreuen staet yuwes selues barmherticheit waert vp dat gy gode behagen mochten want als gy altyt wtghevloyet hebben so solle gy somtijd to $\mathrm{yv}$ seluen gaen vnde mercken yv seluen an wo dat gy van bynen gestalt syn Wo yuwe begherte vnde yuwe wille staet of gy vorwert of achterwert ghaet wo na of wo veer dat gy noch syn den doegheden waer gy $\mathrm{yv}$

(25r) meest in entghaet daghelikes Gy sult yuwe ghebreke vor yuwe oghen halen. vp dat gy yv seluen verwecken moghet to rouwen yvwer sunde vnde sult alle tijd van nyes vp setten $\mathrm{yv}$ seluen to beterne vnde yv seluen sulle gy yv dickewile vnsen leuen heren offeren myt al dat gy vermoghet Vnde hijr af hebbe. wij ene figure in der bibelen wo dat vp ene tijd gedeon de een richter was van israhel stont vnde makede sait schone of reynighede dat van den vncrude do he dit dede do quam de engel godes vnde openbaerde em vnde seghede Dat he solde halen eynen bock vnde solde den seden in water. vnde brenghen dat vleisch vnde dat zap to samen daer he in ghesoden were Vnde so solde he dat vleisch legghen vp enen

(25v) steen den he em wisede vnde solde dan dat sap daer vp gheten Aldus dede gedeon he brochte dat vleisch vnde leghede dat vp den steen vnde ghoet dat sap daer vp Do nam de engel een rode de he in der hant hadde vnde roerde den steen mytter roden do vloith daer eyne vurighe vlamme wt vnde verbrande vleisch vnde sap Wat is vns betekent by gedeon de een richter was van israhel anders dan vnse redelicheit de een richter is geset in vns bynnenste. to ordelne vnde to vnderscheden. Wat is dat he syn coren of sait suuert anders dan dat wij vermyts vnser redelicheit vns pinen van bynnen to vndersoken vnde to doegheden to scheden van den ghebreken. wat is dat de

(26r) engel do gedeon openbaerde Dan dat als wij vns pynen in vns seluen to gane vnde vns to vndersoken dat vns dan in vnsen ver. stande een lecht dat is een wetenheit openbaert de vns vnse ghebreke doet bekennen So spreket de engel dat is dat lecht van bynnen dat vns openbaert vnde seghet hale enen bock vnde sede den in water Dat is neme alle dine vleischelike begheerten vnde sede de in water des rouwen vnde der inwendigher of wtwendigher tranen vnde kom dan vnde legge dat vleisch vp den steen Dat is als du aldus rechten rouwen heuest van dinen sunden so en wil nicht myshopen meer ganck myt rechten betrouwen to vnsen leuen heren ihm xpm de daer seghet Comet to my gy alle de daer belastet syn ouermyts

(26v) manichuoldicheit der cranckheit. vnde der sunde. vnde ic sal yuwe troist wesen vnde weder vermaken want daer vmme hebbe ic den bitteren doet gheleden vp da ic yv van yuwen sunden verlossen mochte Hijr vmme solle wij al de begheerten vnser synne de wij in wtwendigher vleischelicheit. vnde vnser synlicheit plaghen. to ghebruken de solle wij alte male schicken to vnsen leuen heren vp dat wij em behaghen moghen. Vnde nicht allene solle wij vnse begheerte 
dat is vnse buk vp den steen dat is vp xpm legghen Dat is em myt wtwendighen. werken na volghen Meer sij sollen ock dat sap dat syn al vnse ghedachten vnde hope in em setten Vnde als wij aldus

(27r) doen so comet de engel dat is de inwendighe claerheit de vns van bynnen openbaert vnde vns toent vnse sunde vnde ghebreke de roert den steen dat is vnsen leuen heren myt syner roeden ${ }^{74}$ dat is myt ener puerre vnde rechter meninghe vns eweliken van sunden to hoden na vnser macht So springhet een vlamme wt den stene. dat is eyne vurighe mynne to gode vnde to allen doegheden myt enen myshaghen. to vns selues vnde der ghebreken. vnde de verdriuet vnde verbrant. in vns vleischelike begheerte Nicht allene vleischelike werke. meer ock vleischelike ghedachten. dat is dat sap. also dat vns voertmeer nicht en ghenoeghet. Dan wo wij vnsen leuen heren denen moghen vade em vurich-

(27v) liken leifhebben. vnde aldus weerde wij allentelen ouermyts der mynen ouerformet in vnsen leuen heren Also dat wij vns soluen pynen na to volghen dat hillige leuen vnses leuen heren ihu cristi vnde syne passie vnde syne pyne de he van mynnen vmme vnsen willen gheleden heuet in vnsen herten to draghen vnde de ouermyts willigher lijdsamheit na to volghen also langhe als wij leuen vp dat wij myt em leuen vnde regneren moghen in der ewicheit amen Woe be bem oefenen sal van binnen vnde woe be in allen sinen werken syn berte op beuen sal to onsen beren dat ix capittel 75

LEue broder ic hebbe dusse epistel langher ghemaket dan ic ghement hadde to done doch hope ic dattet yv nicht hinderlic. wesen en sal Want den reynen

(28r) vnde puren herten syn alle dinghe reyne vnde puer Meer nv wil ic yv voert scriuen. ene corte oefninghe ${ }^{76}$ daer gy $\mathrm{yv}$ inne oeffenen moghet want gy in vnleden van buten wesen moten Vnde isset dat gy nicht beters of nutters en wetet of al rede hebbet so moghe gy yv daer na regeren isset dat it yv gued dunket. vnde dat it bequeme sy want alle menschen en moghen nicht al ene oefninghe hebben want de naturen. vnde de werke der menschen syn vnghelijc In den eirsten des morghens als gy vp staet so en solle gy yv nicht traechlike noch vakerlic hebben Meer gy sollet yv pynen vlugghe to wesen vnde myt dat gy vp staet of vprichtet so solle gy een cruce vor $\mathrm{yv}$ slaen vnde segghen In den namen vns leuen heren ihu cristi sta ic vp de my verlosset heuet myt synen duer-

(28v) baren blode de mote my huden dussen dach vnde alle daghe regeren benedien bewaren vnde stercken in allen guden werken vnde mote my na dussen vnsalighen leuen brenghen to den ewighen leuen amen Hijr na solle gy vnsen leuen heren dancken dat he yv des nachtes behoit heuet van quade vnde segghen $\mathrm{O}$ almechtighe. ewi-

(74) rod- met bovengeschreven - $\iota$ -

(75) Gekursiveerde titel rood.

(76) -of- met bovengeschreven $-e$ - 
ghe vader ic dancke dy vnde loue dattu vermyts dyner barmherticheit dy gheweerdighet heuest my to behoden in dusser nacht Ic bidde dyner $\mathrm{v} n$ meteliker guderterenheit. dattu my verlenen willest dat ic dussen tocomenden dach myt aller oetmodicheit ${ }^{77}$ gheloue vnde mynnen also ouerbrenghen mote dat dy mijn denst vnde myne werke behaghelic mote wesen ouermyts. vnsen leuen heren ihesum xpm

(29r) amen Daer na moghe gy yuwen engel wat lesen als yv gued duncket Vnde isset dat gy anders wat pleghet to lesen dat moghe gy doen isset so dat gy tijd hebbet Meer gy en sollet yv so seer nicht storten vp vele ghetide. of ghebede to lesen dat gy daer vmme de dinghe de yv beuolen syn to done versumet Of dat yemant daer $\mathrm{v} m \mathrm{me}$ ghebreck of hinder liden solde in den dinghen de he behouede want na den woerden dat de apostel spreket So weert beter enen salmen myt andachte vnde in mynnen ghelesen dan men anders den helen salter lase Vnde als vnse leue here seghet dor den propheten Dit volk eeret my mytten lippen meer oer ${ }^{78}$ herte is veer van my Vnde in den ewangeli Vnde wilt nicht vele spreken in yuwen ghebeden. als de heiden menschen doen

(29v) want yuwe vader in den hemel de weet wat yv noet is Vnde hijr $v m m e$ in manichuoldicheit der ghebeden en wort de mensche nicht ghehoert ${ }^{79}$ van vnsen leuen heren meer in puerheit. des herten vnde der meninghen van bynnen Hijr vmme leue broder solle gy yv meer in den herten pynen to beden vnde yuwe inwendighe begherte. to vnsen leuen heren to pinen to sturen also vele als yv moghelic is na den dat gy vnledich syn van buten Gy sollet yv ock pinen dat gy leert de dinghen de gy van buten hanteert vnde mede vmme gaet dat gy de in wart trecken to dancken vnde to loue vnsen leuen heren Also dat gy seluen dat lof godes syn vnde een voerghaende leuende offerhande vns leuen heren ihu xpi Dat is dat gy yv also pinet to

(30r) hebben dat al yuwe leuen van buten vnde van bynnen gode louet Vnde dat al yuwe beenen dat syn al yuwe inwendighe crachten ropen here we is dyns ghelijc Also dat de ghene de myt yv vmmeghaen vnde yv seen segghen moghen in der waerheit dat gy een knecht godes syn vnde in yuwer oetmodicheit lijdsamheit vnde mynnen gestichtet weerden $G y$ en drouet nicht dencken dat em nemant van bynnen to vnsen leuen heren gheuen mach of verluchtet weerden dan de ghene de in der cellen sitten Want ic hebbet van guden hillighen als ic hope gehoert. vnde ock mach ment in synen scriften lesen dat rusebrokes koc de nochtan vijf ammete hadde to verwaren dat was de koeken de gaste. backen brouwen vnde

(30v) dat he dat holt moste halen wt den bussche dat men plach to bezeghen vnde ock was he als he seluen seghet vnde ock deghene tughen

(77) ot- met bovengeschreven -e-.

(78) or- met bovengeschreven - $e$ -

(79) -hort met bovengeschreven $-e$ - 
diet weten een grof mensche van buten vnde een puer leye dusse hadde in syner inwendigher. vnleden meer inwendighes gheuolens vnde gotlikes luchtes dan somyghe hundert monike of clerke hebben de nochtan in der cellen sitten vnde alle reeschop daer to hebben Vnde hijr vmme leue broder pinet yv to done dat in yv vnde laet voert ${ }^{80}$ vnsen leuen heren myt yv beghaen Hijr na moghe gy isset dat gy daer nicht vp ghedacht en hebben dencken wat gy to done hebbet vp dat gy nicht en versumet de dinghen de yv beuolen syn want dat gy doen sult van ghehoersamheit to den loue vnde to der ere godes dat sal

(31r) alletijd voerghaen Voert wanneer gy of clemmet van ywer camer daer gy slapet so moghe gy dencken isset dat gy willet wo dat Jacob de patriarche de engelen sach vp vnde neder clemmen vnde vnsen leuen heren vp dat ouerste der ledderen rasten Vnde dan solle in yuwen herten begheren dat vnse leue here gheweerdighe van syner guetheit syne hilligen engele neder to senden vp dat se yv vnde alle syne vercoren regeren sturen vnde stercken moghen in synen rechten weghe vnde in allen guden werken Hijr na als gy vuer maken sollet vmme yvwe spise to reden so moghe gy dencken wo dat vnse leue here vp den pinxte dach den hilligen geist sande in ghelikenisse des vures in syne apostolen vnde wtuercoren vade dan solle gy em yn yuwen herten bidden vnsen leuen heren

(31v) dat he yv vnde alle syne vercorene entsteken wille mytten vure syner mynnen also dat he van em vurichlike. ghemynt vnde ghelouet weerde in tijd vnde in ewicheit Voert als gy to den putte ghaet vmme water to putten so solle gy versuchten vnde claghen in yuwe dorricheit vnde lauheit. van bynnen vnde dat vnse leue here in manighen menschen so luttinck gheachtet vnde bekant is $\mathrm{Vnde}$ dan solle gy myt oetmodighen ${ }^{81}$ vnde mynliken herten myt inwendighen medeliden begheren van vnsen leuen heren dat he yuwe dorre herte vnde al synre ghemeynder begheren wille vnde nat maken myt water syner gracien van bouen Vnde mytten douwe syner inwendigher ghenaden. vnde synen hillighen in em sende vnde in em make eene leuende fonteyne. springhende in dat ewighe leuen

(32r) vp dat syn hillige name daer af ghelouet vnde gheert weerde in der ewicheit Hijr na als gy de vate nemet daer gy de spise in reden sollet so solle vnsen leuen mynliken heren dancken vnde louen Dat he vns van allen dinghen so mynliken vnde so guderterelike vorseen heuet vnde versuet de vns nottruftich syn Vnde dan solle gy spreken in yuwen herten vade segghen $\mathrm{O}$ mynlike vnde guderterene. here ic bidde dy dor dyne vnsprekelike. barmherticheit dattu alle menschen dat dyne verstantlike vnde redelike vate syn de du mynlike here daer to gheschapen heuest vp dat se bequame vnde nutte solden wesen vp dattu dyne gracie vnde ghauen daer in storten mochtes de anders nicht en begheers Dan dattu ledighe. vnde

(80) vort met bovengeschreven $-e$ -

(81) of- met bovengeschreven $-e$-. 
reyne vate vondes dyne

$(32 v)$ ghenade in to storten. want du van dyner vnsprekeliker myldicheit altijt rede bist to gheuen waert datter bequame vate waren deit entfanghen. mochten Vnde dy mynlike here en is nicht ghenoech dattu dyne ouervlodighe. ghauen daer in stortest. meer dicwillest seluen daer in wonen als in dynen leuenden vnde redeliken tempel Hijr vmme mynlike here com vnde vervulle de herten dyner ghemynder myt dy seluen. vp dat se dy louen dancken vnde benedien moghen vermyts dy seluen in tijd vnde in ewicheit Voert wanneer ${ }^{82}$ dat gy de potte roret so moghe gy dencken wo de mynne oer alle dinghe ghemene maket $\mathrm{Vnde}$ dan solle gy begheren van vnsen leuen heren dat he yv late ghemeenheit hebben vnde deylaftich weerden syner verdensten vnde al der hilliger ghe-

(33r) meenheit. der hillighen kerken Wanneer gy de spise schumet so moghe gy dencken wo dat dat vule vnde quade altijt in vns bouen comet vnde dat wij altijt moten af werpen vnde schone maken solle wij bequeme spise weerden in vnsen leuen heren to ghangen Vnde gu sollet em bidden dat he dat vuer der mynnen in yw entsteke mytten lechte syner waerheit vp dat gy yv seluen vnde yuwe ghebreke bekennen moghen vnde yv vermyts syner ghenade daer af suueren vnde reyne maken also dat gy em behaghen. moghen Voert wanner gy merket vnde anseet de torue vnde dat holt bernen in den vure so moghe gy dencken wo de sele ligghen vnde bernen in der helle vade in den veghevuer. vnde yv seluen verwecken to penitencien. vnde berouwe yuwer sunden Vnde

(33v) moghet dencken datter clene vnde luttinck is dat men hijr liden mach daer men dat ewighe vuer mede entghaet vnde de ewighe blijtschop vercrighet. vnde aldus solle gy $\mathrm{yv}$ seluen verwecken to guden werken der mynnen vnde yv de nicht laten verdreten Vnde voert solle gy merkelike medeliden hebben mytten ghenen de in den veghevuer. syn vnde daer voer truwelike. bidden. vnde begheren myt rechten dat gy gheerne al yuwen arbeyt em willet to bate comen laten vp dat se verlost mochten weerden van der pinen. vnde to em comen daer se to gheschapen syn Hijr na als gy de spise vp richten sollet so moge gy begheren in yuwen herten vnde segghen. $\mathrm{O}$ guderterene vnde mynlike here de vermyts dyner vnsprekeliker myldicheit alle creaturen in hemel

(34r) vnde in eerden vervullest myt dyner benediccien vnde gotliken ghauen come vnde vervulle de bynnensten dyner knechten mytten lechte dyner waerheit. vp dat se nicht allene myt dusser spisen van buten ghevoet. vnde ghesterket moten weerden meer ock myt al den hilligen woerden ${ }^{83}$ de wt dynen monde ghaen verluchtet. vnde ghesterket moghen weerden vnde dy de dat hemelsche broet bist in der ewicheit ghebruken moghen Voert als gy de potte vnde schottelen wasschet so moghe gy vnsen leuen heren dancken dat he vns in allen

(82) wanner met bovengeschreven -e-.

(83) word- met bovengeschreven -e- 
dinghen besorghet heuet vnde heuet vns ghegheuen dat element des waters. to vnsen orbaer des wij nicht. entberen en mochten want wij daer inne vns vnde ander dinghe van buten mede reynighen mogen

(34v) Vnde biddet em oetmodelike dat he van syner ewigher guetheit yv herten vnde al syner ghemynder verlenen wille warachtich berou vnde vurighe. beweghinge to hebben van bynnen vp dat se vermyts den water der inwendigher vnde wtwendigher tranen ghewasschen moghen weerden van eren sunden vnde also bequame vnde reyne vate weerden als dat he syne gracie vnde ghenade daer in storten moghe to synen loue vnde to syner ere Voert ${ }^{84}$ wanneer dat gy beesten of vische dodet of ter kocken houwet vnde bereyt so moghe gy altijt vnsen leuen heren dancken dat he den visch of dat vleisch to vnser noetruft ghemaket heuet vnde vns dat verseen van ewicheit Meer dit moghe gy ock in yv seluen trecken vnde dencken wo dat beesten vnde

(35r) de vissche de hijr eren vryen ganck hebben vnde na erer ghenoichte leuen de moten toer ${ }^{85}$ koeken ghehouwen weerden vnde betalen dat se gheten hebben Also isset mytten menschen de hijr al er ghenoechte willen bruken vnde leuen na den wtwendighen synnen als een beest. de sollen vnverseenlic. van der doet gheslaghen weerden. vnde van den helschen kocken dat sijn de duuelen to den ewighen vure ghebracht weerden daer se ghebraden vnde ghesoden sollen weerden sunder ende $\mathrm{Vnde}$ dit moghe gy to $\mathrm{yv}$ seluen trecken vp dat yy nicht en verdrete ene corte tijt vmme vns leuen heren willen to arbeyden want it en sal nicht langhe duren $\mathrm{Hijr}$ na als gy to mysse gaet so moghe gy myt ynnighen herten als gy best moghet dencken wat de mysse be-

(35v) dudet vnde waer vmme se ghesat is to done dat is in ghehoichnissen der eirsten mysse de vnse mynlike here ihesus xps vp den lesten auentmale do he syn hillighe licham sijn vleisch vnde syn bloet ghaf synen discipulen unde em seluen daer na offerde eene ghename offerhande synen ewighen vader vp den altaer des cruces $\mathrm{V}$ de so moghe in der mysse. een punt of twe van syner mynliker. passien voernemen vnde daer vp dencken is dat gy nicht beters en wetet of sunderlinghe to lesen en hebbet Voert wanneer gy hoert dat yemant cloppet vmme yv to hebben of to spreken so solle gy myt mynliken. herten dencken dat is de stemme mijns ghemynden vnde sollet dan oetmodelike comen vnde em to spreken. recht of gy vnsen leuen heren

(36r) in syns selues persoen voer yv hadden vnde myt em spreken Leue broder dusse dinghe hebbe ic gheschreuen al syn se plump unde grof. vp dat gy in allen dinghen yv solden pinen $\mathrm{yv}$ beste to soiken vnde yv also wennen dat gy in allen dinghen de gy van buten han-

(84) Vort met bovengeschreven - $e$ -

(85) tor met bovengeschreven -e-. 
teert. gode schouwen vnde voer ${ }^{86}$ oghen hebben moghen Voert ${ }^{87}$ solle gy alle tijd yuwe inwendighe oghen slaen vp dat soite vnde mynlike leuen vnses leuen heren ihu xpi wo mynlic vnde wo oetmodelic vnde wo lidesamlic he em altijd hadde myt synen discipulen. vnde myt den ghenen daer he mede vmme ghenck Also dat he nicht versmaet en heuet de voete to wasschen der armen visschers vnde heuet em oetmodelike ghedeent als een knecht Hijr na des auendes als gy slapen ghaet is dat gy moede

(36v) syn vide vele ghearbeydet hebbet so en solle gy yv nicht to vele pynen to lesen meer gy sollet yv pynen myt corten ghebeden to bedde to ghane vp dat gy des morghens to wackerre moghet wesen de dinghen. of de wercke der ghehoersamheit to done de yv beuolen syn want vnse leue here en gheuet vmme vele lesen nicht als voer ghescreuen is meer dat gy vele guder werke der mynnen vnde der ghehoersamheit werken Vnde hijr af lese wij een exempel van sunte berndes broders. Wo dat was een rekelic convers. de des daghes vele ghearbeydet. hadde in wtwendighen arbeide also dat he vermyts swaerheit vnde manichuoldicheit des arbeides syne sunderlinghe ghebede de he daghelikes plach to lesen nicht

(37r) ghelesen en hadde des auendes do he slapen solde ghaen do pinede he em dat he verhalen wolde dat he vermyts den arbeyde des daghes versumet hadde meer wat he pinede et was al vmme nicht. he en conde syne dinghe nicht ghedoen noch ghelesen als he gheerne ghedaen hadde Also dat he merkelike daer inne beswaert vnde bedrouet waert Do quam een stemme vnde sprack em to vnde seghede wat wultu arbeiden. vmme dijn ghebet to lesen lete ic des nicht vmme dijns abbates willen ic solde dy wt den synen laten comen wat hebbe ic myt dynen beden to done ic hebbe leuer ghehoersamheit. dan offerhande Daer vmme ghanck slapen vp dattu morghen starker bist de werke der ghehorsamheit to done de dy be-

(37v) uolen. weerden Want de ghehoersamheit. vervullet wat dy anders enbrecket Vnde hijr vmme en pynet yv des auendes als gy mode synt nicht vele to lesen daer gy nicht to verbunden en syn. meer dat gy wilt so moghe gij hijr mede slapen ghaen dat hijr na ghescheuen staet vnde segghen $\mathrm{O}$ mynlike here vnde hillige vader $\mathrm{O}$ almechtighe vnde ewige god ic bidde dy dor dyne ewighe guetheit. wille my barmhertelike. ouermyts de verwynninghe des hillighen cruces wat ic in dussen. daghe mysdaen hebbe vnde my entgaen ouermyts versumelheit in woerden in werken vnde in ghedachten. guderterelike willest vergheuen Vnde wille my voert ${ }^{88}$ van aller anvechtinghe vnde fantasien des duuels. in dusser nacht behoden

(38r) vp dat ic vermyts dyner gracien vnde ghenaden van aller duveliker vnde menscheliker bedreichnisse van dyner guetheit beschermet mach weerden vnde des morghens myt gotliker vrouden vade blijtschop vp staen moghe. vmme de vnsprekelike moghentheit. dyns hilligen

(86) vor met bovengeschreven $-e$ -

(87) Vort met bovengeschreven -e-

(88) vort met bovengeschreven $-e$ - 
namen to dancken vnde to louen Ouermyts vnsen leuen heren ihm xpm amen Hijr na solle gy em dancken dat he yv des daghes behod vnde beschermet heuet vnde segghen. $\mathrm{O}$ here vnde hillige vader ic dancke. dy myt al mynen herten van dat dy my ouermyts dyner vnsprekeliker guderterenheit gheweerdighet heues to behoden vnde to beschermen in dussen. daghe wille my mynlike here verlenen. dusse tocomende nacht myt reynen herten vnde lichame also mer to brenghen als wanneer ic des morghens

(38v) vp sta dy mynen mynliken heren louen vnde dancken moghe als ic schuldich. byn to done Ouermyts vnsen leuen heren ihm xpm dynen sone de mytti leuet vnde regneret in ewicheit des hilligen gheistes een god in ewicheit amen Wille gy dan yuwen engel wat lesen dat hope ic dat gued is. vnde aldus moghe gy slapen ghaen vnde offeren yuwen gheist vnde yuwen licham in den handen vns ewighen vaders. de vns vermyts synen ewighen sone verloset heuet van den ewighen dode Leue broder ic mochte yv vele meer scriuen hadde ic de wijsheit vnde dattet my vnse mynlike here ghaue doch so hope ic dattet wal liden mach Meer dat ende van allen guden oefninghen vnde van alre gheistelicheit Dat is dat gy $\mathrm{yv}$ al heel ouer coent gheuen

(39r) vnsen leuen heren vnde yuwen ouersten dat he myt yv doen moghe wat he wil Vnde dat gy yv pinet in al yuwen werken van bynnen vnde van buten meer to soiken vnde to nemen dat lof vnde de ere godes dan yuwes selues nutticheit of orbaer hijr of in der ewicheit Hijr vmme laet vns to samen bidden vnsen leuen heren dat wij dit clene vulbrenghen moten vnde also to den meesten vnde den hoechsten ghereken ouermyts vnsen leuen heren ihesum xpm de mytten vader vnde mytten hillighen geiste eyn god is in ewicheit

Wo een guetwillich koc in den eirsten synen staet an merken sal vnde em pinen den to ordineren to der meyster ere godes dat eirste capittel

Wo he em sunderlinghe setten moet

(39v) to lijdsamheit vnde to sachtmodicheit. ij capittel. Wo he em hebben sal in der yeghenwordicheit sijns ouersten vnde syner broders .iij. We he em hebben sal in synen becoringhen. iiij. Wo dat beghin nicht allene an to seen is meer dat men vulherde to in den ende .v. Wo dat he nicht beswaert en sal wesen als em dorre vnde vnbegheerlic gheuoelt. vnde wat waer deuocie is vj Wo he em hebben sal to den seken vnde de syner to doende hebten vij Wo dai men altijd nicht verluchtet en wert de scrift to lesen vnde to horen. meer als men werke der mynnen doet. viij.

Wo he em oeffenen sal van bynnen vnde wo he in al synen werken syn herte vp heffenen sal to vnsen heren ix capittel.

Ghescreuen Int iaer vns heren dusent $\mathrm{cccc}^{\circ}$ vnde .lv. 